\title{
Multi-dimensional BSDEs driven by $G$-Brownian motion and related system of fully nonlinear PDEs
}

\author{
Guomin Liu *
}

\begin{abstract}
In this paper, we study the well-posedness of multi-dimensional backward stochastic differential equations driven by $G$-Brownian motion ( $G$-BSDEs). The existence and uniqueness of solutions are obtained via a contraction argument for $Y$ component and a backward iteration of local solutions. Furthermore, we show that, the solution of multi-dimensional $G$-BSDE in a Markovian framework provides a probabilistic formula for the viscosity solution of a system of nonlinear parabolic partial differential equations.
\end{abstract}

Key words: $G$-expectation, $G$-Brownian motion, Multi-dimensional BSDEs, Fully nonlinear PDEs.

MSC-classification: $60 \mathrm{H} 10,60 \mathrm{H} 30$.

\section{Introduction}

The nonlinear backward stochastic differential equation driven by Brownian motion (BSDE) was introduced by Pardoux and Peng [13. A solution to a BSDE is a couple of processes $(Y, Z)$ satisfying

$$
Y_{t}=\xi+\int_{t}^{T} f\left(s, Y_{s}, Z_{s}\right) d s-\int_{t}^{T} Z_{s} d B_{s}, 0 \leq t \leq T,
$$

where $B_{t}$ is a $d$-dimensional standard Brownian motion and the function $f$ is called the generator of BSDE. Since then, it was shown that the BSDE theory is a powerful tool for the study of partial differential equations, stochastic control, theoretical economics and mathematical finance, see [2, 4, 6, 17.

Recently, motivated by financial problems with Knightian uncertainty, Peng [18, 19, 20] has established systematically a framework of time-consistent sublinear expectation, called $G$-expectation. Under this framework, a new type of nonlinear Brownian motion $\left(B_{t}\right)_{t \geq 0}$ called $G$-Brownian motion and the corresponding stochastic calculus were established. Furthermore, Hu, Ji, Peng and Song [7] obtained the existence and uniqueness of solutions to the following one-dimensional (i.e., $Y$ is onedimensional) backward stochastic differential equations driven by $G$-Brownian motion ( $G$-BSDEs):

$$
Y_{t}=\xi+\int_{t}^{T} f\left(s, Y_{s}, Z_{s}\right) d s+\int_{t}^{T} g_{i j}\left(s, Y_{s}, Z_{s}\right) d\left\langle B^{i}, B^{j}\right\rangle_{s}-\int_{t}^{T} Z_{s} d B_{s}-\left(K_{T}-K_{t}\right),
$$

where the solution of this equation consists of a triple of processes $(Y, Z, K)$. Note that, compared with the classical case, $G$-BSDE has an extra non-increasing $G$-martingale term $K$. In their accompanying

\footnotetext{
${ }^{*}$ School of Mathematical Sciences, Fudan University, Shanghai, 200433, PR China; and Zhongtai Securities Institute for Financial Studies, Shandong University, Jinan, 250100, PR China. gmliusdu@163.com. Research supported by the National Natural Science Foundation of China (No. 11601282 and No.11671231) and the Natural Science Foundation of Shandong Province (No. ZR2016AQ10).
} 
paper [8], the corresponding comparison theorem, Feymann-Kac formula were established. For other developments, one can refer to, for instance, [10, 12, 23. See also [21] for a formulation highly related to $G$-BSDEs, called 2BSDEs.

Multi-dimensional $G$-BSDE refers to the case that $Y$ in the solution is multi-dimensional. Since $G$ expectation is a nonlinear expectation, the linear combination of $G$-martingales is not a $G$-martingale anymore. This leads to the difficulty that the method for one-dimensional $G$-BSDE in 7 can not be applied to solving multi-dimensional $G$-BSDEs.

We denote by $x^{l}$ the $l$-th column (component) of $x \in \mathbb{R}^{n}$, for $l=1, \cdots, n$. In the paper, we consider the following multi-dimensional $G$-BSDEs:

$$
Y_{t}^{l}=\xi^{l}+\int_{t}^{T} f^{l}\left(s, Y_{s}, Z_{s}^{l}\right) d s+\int_{t}^{T} g_{i j}^{l}\left(s, Y_{s}, Z_{s}^{l}\right) d\left\langle B^{i}, B^{j}\right\rangle_{s}-\int_{t}^{T} Z_{s}^{l} d B_{s}-\left(K_{T}^{l}-K_{t}^{l}\right), \quad 1 \leq l \leq n .
$$

Here the generators $f, g_{i j}$ are assumed to be diagonal in $z$, i.e., the $z$ parts of the $l$-th components $f^{l}, g_{i j}^{l}$ only depend on $z^{l}$. The existence and uniqueness theorem (see Theorem 3.1) of the local solution to (2) is proved by the method of Picard iteration for the $Y$ term, in which we have applied the well-posedness theorem of one-dimensional $G$-BSDEs and the tools of a priori estimates. The global solution (see Theorem 3.5) is then obtained by a backward iteration of the local ones. The Picard iteration for local solutions is also used to establish the comparison theorem (see Theorem 3.6) for multi-dimensional $G$-BSDE (2) through a limit argument.

In the last part of our paper, we study the link with the viscosity solution of a system of fully nonlinear parabolic partial differential equations in a Markovian framework. Indeed, 8 showed that the one-dimensional $G$-BSDE is connected to fully nonlinear parabolic partial differential equation. On the other hand, the solution to a standard multi-dimensional BSDE provides the viscosity solution to a system of semilinear parabolic partial differential equations, see, e.g., Pardoux and Peng 14 Pardoux, Pradeilles and Rao 15, Peng [16. Inspired by these two directions, we show that, our multi-dimensional $G$-BSDE can provide a probabilistic representation for the solution of a system of fully nonlinear parabolic partial differential equations (see Theorem 4.7). For this purpose, we derive some useful a priori estimates of continuous dependence of solutions on terminal conditions and generators for $G$-BSDEs (2), and also study the regularity of solutions of $G$-BSDEs (2) coupled with stochastic differential equations driven by $G$-Brownian motion $(G$-SDEs).

This paper is organized as follows. In Section 2, we recall some basic notions of $G$-Brownian motion and one-dimensional $G$-BSDEs. Section 3 is devoted to the well-posedness of multi-dimensional $G$ BSDEs and the corresponding comparison theorem. Finally, in Section 4, we give its relationship with the system of PDEs.

\section{Preliminaries}

The main purpose of this section is to recall some basic results about $G$-expectation and onedimensional $G$-BSDEs, which are needed in the sequel. The readers may refer to [5, 7, 8, 20, for more details. For convenience, every element $x \in \mathbb{R}^{n}$ is identified to a column vector with $l$-th component $x^{l}$ and the corresponding Euclidian norm and Euclidian scalar product is denoted by $|\cdot|$ and $\langle\cdot, \cdot\rangle$, respectively.

\section{$2.1 \quad G$-expectation}

Let $\Omega_{T}:=C_{0}\left([0, T] ; \mathbb{R}^{d}\right)$ be the space of all $\mathbb{R}^{d}$-valued continuous paths $\left(\omega_{t}\right)_{t \geq 0}$ starting from origin, equipped with the supremum norm. Denote by $\mathcal{B}\left(\Omega_{T}\right)$ the Borel $\sigma$-algebra of $\Omega_{T}$ and $B_{t}(\omega):=\omega_{t}$ the canonical mapping. For each $t \in[0, T]$, we set

$$
L_{i p}\left(\Omega_{T}\right):=\left\{\varphi\left(B_{t_{1}}, \ldots, B_{t_{k}}\right): k \in \mathbb{N}, t_{1}, \ldots, t_{k} \in[0, T], \varphi \in C_{b . L i p}\left(\mathbb{R}^{k \times d}\right)\right\},
$$


where $C_{b . L i p}\left(\mathbb{R}^{k \times d}\right)$ denotes the space of bounded and Lipschitz functions on $\mathbb{R}^{k \times d}$.

Let $\mathbb{S}(d)$ be the space of all $d \times d$ symmetric matrices. For each given monotonic and sublinear function $G: \mathbb{S}(d) \rightarrow \mathbb{R}$, Peng [20] constructed a dynamic coherent nonlinear $G$-expectation space $\left(\Omega_{T}, L_{i p}\left(\Omega_{T}\right), \hat{\mathbb{E}},\left(\hat{\mathbb{E}}_{t}\right)_{t \geq 0}\right)$. In this paper, we always assume that $G$ is non-degenerate, i.e., there exists a constant $\underline{\sigma}^{2}>0$ such that

$$
G(A)-G(B) \geq \frac{1}{2} \underline{\sigma}^{2} \operatorname{tr}[A-B], \text { for } A \geq B .
$$

For each $p \geq 1$, the completion of $L_{i p}\left(\Omega_{T}\right)$ under the norm $\|X\|_{L_{G}^{p}}:=\left(\hat{\mathbb{E}}\left[|X|^{p}\right]\right)^{1 / p}$ is denoted by $L_{G}^{p}\left(\Omega_{T}\right)$. The $G$-expectation $\hat{\mathbb{E}}[\cdot]$ and conditional $G$-expectation $\hat{\mathbb{E}}_{t}[\cdot]$ can be extended continuously to the completion $L_{G}^{p}\left(\Omega_{T}\right)$. The corresponding canonical process $B$ is called $G$-Brownian motion.

Indeed, the $G$-expectation can be regarded as an upper expectation on $L_{G}^{1}\left(\Omega_{T}\right)$.

Theorem $2.1([\mathbf{5}, \mathbf{9}])$ There exists a weakly compact set $\mathcal{P}$ of probability measures on $\left(\Omega_{T}, \mathcal{B}\left(\Omega_{T}\right)\right)$ such that

$$
\hat{\mathbb{E}}[\xi]=\sup _{P \in \mathcal{P}} E_{P}[\xi]=\max _{P \in \mathcal{P}} E_{P}[\xi], \quad \text { for all } \xi \in L_{G}^{1}\left(\Omega_{T}\right) .
$$

For this $\mathcal{P}$, we define capacity

$$
c(A):=\sup _{P \in \mathcal{P}} P(A), A \in \mathcal{B}\left(\Omega_{T}\right) .
$$

A set $A \subset \Omega_{T}$ is polar if $c(A)=0$. A property holds "quasi-surely" (q.s. for short) if it holds outside a polar set. In the following, we do not distinguish between two random variables $X$ and $Y$ if $X=Y$ q.s..

For $p \geq 1$, we set

$$
\mathbb{L}^{p}\left(\Omega_{T}\right):=\left\{X \in \mathcal{B}\left(\Omega_{T}\right): \sup _{P \in \mathcal{P}} E_{P}\left[|X|^{p}\right]<\infty\right\} .
$$

It is important to note that $L_{G}^{p}\left(\Omega_{T}\right) \subset \mathbb{L}^{p}\left(\Omega_{T}\right)$. We extend $G$-expectation $\hat{\mathbb{E}}$ to $\mathbb{L}^{p}\left(\Omega_{T}\right)$, and still denote it by $\hat{\mathbb{E}}$, by setting

$$
\hat{\mathbb{E}}[X]:=\sup _{P \in \mathcal{P}} E_{P}[X], \text { for each } X \in \mathbb{L}^{1}\left(\Omega_{T}\right) .
$$

For $p \geq 1, \mathbb{L}^{p}\left(\Omega_{T}\right)$ is a Banach space under the norm $\left(\hat{\mathbb{E}}\left[|\cdot|^{p}\right]\right)^{1 / p}$.

In the sequel, we shall also use the $G$-evaluation introduced in 22 . For $\xi \in L_{i p}\left(\Omega_{T}\right)$, we define its $G$-evaluation by $\mathcal{E}[\xi]:=\hat{\mathbb{E}}\left[\sup _{t \in[0, T]} \hat{\mathbb{E}}_{t}[\xi]\right]$. For $p \geq 1$ and $\xi \in L_{i p}\left(\Omega_{T}\right)$, define $\|\xi\|_{p, \mathcal{E}}=\left\{\mathcal{E}\left[|\xi|^{p}\right]\right\}^{1 / p}$ and denote by $L_{\mathcal{E}}^{p}\left(\Omega_{T}\right)$ the completion of $L_{i p}\left(\Omega_{T}\right)$ under the norm $\|\cdot\|_{p, \mathcal{E}}$.

Theorem 2.2 For any $\alpha \geq 1$ and $\delta>0$, we have $L_{G}^{\alpha+\delta}\left(\Omega_{T}\right) \subset L_{\mathcal{E}}^{\alpha}\left(\Omega_{T}\right)$. More precisely, for all $\xi \in L_{i p}\left(\Omega_{T}\right)$, we have

$$
\hat{\mathbb{E}}\left[\sup _{t \in[0, T]} \hat{\mathbb{E}}_{t}\left[|\xi|^{\alpha}\right]\right] \leq C\left(\hat{\mathbb{E}}\left[|\xi|^{\alpha+\delta}\right]\right)^{\alpha /(\alpha+\delta)},
$$

for some constant $C$ depending only on $\alpha$ and $\delta$.

Remark 2.3 In [22, the author gives the following inequality for the estimate of $G$-evaluation: there exists some constant $C$ depending only on $\alpha$ and $\delta$ such that

$$
\hat{\mathbb{E}}\left[\sup _{t \in[0, T]} \hat{\mathbb{E}}_{t}\left[|\xi|^{\alpha}\right]\right] \leq C\left\{\left(\hat{\mathbb{E}}\left[|\xi|^{\alpha+\delta}\right]\right)^{\alpha /(\alpha+\delta)}+\hat{\mathbb{E}}\left[|\xi|^{\alpha+\delta}\right]\right\} .
$$

The refree points out that the above inequality can be refined to be (3), which is more similar to the classical case. Indeed, multiplying $\xi$ by $\lambda>0$ and making $\lambda$ tend to 0 , then the nonhomogeneous term is eliminated and we get the stronger inequality (3). 


\subsection{One-dimensional $G$-BSDEs}

First, we shall introduce the corresponding stochastic calculus with respect to $G$-Brownian motion.

Definition 2.4 Let $M_{G}^{0}(0, T)$ be the collection of processes in the following form: for a given partition $\left\{t_{0}, \cdots, t_{N}\right\}$ of $[0, T]$,

$$
\eta_{t}(\omega)=\sum_{j=0}^{N-1} \xi_{j}(\omega) I_{\left[t_{j}, t_{j+1}\right)}(t)
$$

where $\xi_{i} \in L_{i p}\left(\Omega_{t_{i}}\right), i=0,1,2, \cdots, N-1$. For $p \geq 1$ and $\eta \in M_{G}^{0}(0, T)$, let $\|\eta\|_{H_{G}^{p}}=\left\{\hat{\mathbb{E}}\left[\left(\int_{0}^{T}\left|\eta_{s}\right|^{2} d s\right)^{p / 2}\right]\right\}^{1 / p}$, $\|\eta\|_{M_{G}^{p}}=\left\{\hat{\mathbb{E}}\left[\int_{0}^{T}\left|\eta_{s}\right|^{p} d s\right]\right\}^{1 / p}$ and denote by $H_{G}^{p}(0, T), M_{G}^{p}(0, T)$ the completions of $M_{G}^{0}(0, T)$ under the norms $\|\cdot\|_{H_{G}^{p}},\|\cdot\|_{M_{G}^{p}}$ respectively.

For each $1 \leq i, j \leq d$, we denote by $\left\langle B^{i}, B^{j}\right\rangle$ the mutual variation process. Then for two processes $\eta \in H_{G}^{p}(0, T)$ and $\xi \in M_{G}^{p}(0, T)$ with $p \geq 1$, the $G$-Itô integrals $\int_{0}^{\cdot} \eta_{s} d B_{s}^{i}$ and $\int_{0}^{\cdot} \xi_{s} d\left\langle B^{i}, B^{j}\right\rangle_{s}$ are well defined, see [7.

Now we introduce some basic results of one-dimensional $G$-BSDEs. Consider the following type of $G$-BSDEs (in this paper we always use Einstein's summation convention)

$$
Y_{t}=\xi+\int_{t}^{T} f\left(s, Y_{s}, Z_{s}\right) d s+\int_{t}^{T} g_{i j}\left(s, Y_{s}, Z_{s}\right) d\left\langle B^{i}, B^{j}\right\rangle_{s}-\int_{t}^{T} Z_{s} d B_{s}-\left(K_{T}-K_{t}\right),
$$

where

$$
f(t, \omega, y, z), g_{i j}(t, \omega, y, z):[0, T] \times \Omega_{T} \times \mathbb{R} \times \mathbb{R}^{d} \rightarrow \mathbb{R}
$$

satisfy the following properties:

$\left(A_{1}\right)$ there exists some $\beta>1$ such that for any $y, z, f(\cdot, \cdot, y, z), g_{i j}(\cdot, \cdot, y, z) \in M_{G}^{\beta}(0, T)$,

$\left(A_{2}\right)$ there exists some $L>0$ such that

$$
\left|f(t, y, z)-f\left(t, y^{\prime}, z^{\prime}\right)\right|+\sum_{i, j=1}^{d}\left|g_{i j}(t, y, z)-g_{i j}\left(t, y^{\prime}, z^{\prime}\right)\right| \leq L\left(\left|y-y^{\prime}\right|+\left|z-z^{\prime}\right|\right)
$$

Let $S_{G}^{0}(0, T)=\left\{h\left(t, B_{t_{1} \wedge t}, \ldots, B_{t_{n} \wedge t}\right): t_{1}, \ldots, t_{n} \in[0, T], h \in C_{b, L i p}\left(\mathbb{R}^{n+1}\right)\right\}$. For $p \geq 1$ and $\eta \in S_{G}^{0}(0, T)$, set $\|\eta\|_{S_{G}^{p}}=\left\{\hat{\mathbb{E}}\left[\sup _{t \in[0, T]}\left|\eta_{t}\right|^{p}\right]\right\}^{1 / p}$. Denote by $S_{G}^{p}(0, T)$ the completion of $S_{G}^{0}(0, T)$ under the norm $\|\cdot\|_{S_{G}^{p}}$. For simplicity, we denote by $\mathcal{A}_{G}^{p}(0, T)$ the collection of processes $K$ such that $K$ is a non-increasing $G$-martingale with $K_{0}=0$ and $K_{T} \in L_{G}^{p}\left(\Omega_{T}\right)$.

In the rest of this paper, for simplicity we denote by $M_{G}^{p}\left(0, T ; \mathbb{R}^{n}\right)$ the set of $n$-dimensional stochastic process $X=\left(X^{1}, \cdots, X^{n}\right)$ such that $X^{l} \in M_{G}^{p}(0, T), l \leq n$, and we also define $S_{G}^{\alpha}\left(0, T ; \mathbb{R}^{n}\right)$, $H_{G}^{\alpha}\left(0, T ; \mathbb{R}^{n \times d}\right), \mathcal{A}_{G}^{\alpha}\left(0, T ; \mathbb{R}^{n}\right)$ and $L_{G}^{\beta}\left(\Omega_{T} ; \mathbb{R}^{n}\right)$ similarly.

Theorem 2.5 (Theorem 4.1 of [7]) Assume that $\xi \in L_{G}^{\beta}\left(\Omega_{T}\right)$ and $f, g_{i j}$ satisfy $\left(A_{1}\right)$ - $\left(A_{2}\right)$ for some $\beta>1$. Then for any $1<\alpha<\beta$, equation (4) has a unique solution $(Y, Z, K) \in S_{G}^{\alpha}(0, T) \times$ $H_{G}^{\alpha}\left(0, T ; \mathbb{R}^{d}\right) \times \mathcal{A}_{G}^{\alpha}(0, T)$.

Theorem 2.6 (Comparison theorem ([8])) Let $\left(Y_{t}^{(l)}, Z_{t}^{(l)}, K_{t}^{(l)}\right)_{t \leq T}, l=1,2$, be the solutions of the following $G$-BSDEs:

$$
Y_{t}^{(l)}=\xi^{(l)}+\int_{t}^{T} f^{(l)}\left(s, Y_{s}^{(l)}, Z_{s}^{(l)}\right) d s+\int_{t}^{T} g_{i j}^{(l)}\left(s, Y_{s}^{(l)}, Z_{s}^{(l)}\right) d\left\langle B^{i}, B^{j}\right\rangle_{s}-\int_{t}^{T} Z_{s}^{(l)} d B_{s}-\left(K_{T}^{(l)}-K_{t}^{(l)}\right),
$$


where $f^{(l)}, g_{i j}^{(l)}$ satisfy $\left(A_{1}\right)-\left(A_{2}\right)$ and $\xi^{(l)} \in L_{G}^{\beta}\left(\Omega_{T}\right)$ with $\beta>1$. If $\xi^{(1)} \geq \xi^{(2)}, f^{(1)} \geq f^{(2)},\left[g_{i j}^{(1)}\right]_{i, j=1}^{d} \geq$ $\left[g_{i j}^{(2)}\right]_{i, j=1}^{d}$ (here $\left[g_{i j}^{(k)}\right]_{i, j=1}^{d}$ stands for $d \times d$ matrix with entries $g_{i j}^{(k)}, k=1,2$, and $\left[g_{i j}^{(1)}\right]_{i, j=1}^{d} \geq\left[g_{i j}^{(2)}\right]_{i, j=1}^{d}$ means that $\left[g_{i j}^{(1)}\right]_{i, j=1}^{d}-\left[g_{i j}^{(2)}\right]_{i, j=1}^{d}$ is nonnegative definite), then $Y_{t}^{(1)} \geq Y_{t}^{(2)}$.

We also have the following estimates.

Proposition 2.7 (Propositions 3.8 and 5.1 of [7]) For each $T \leq S$, let $\xi^{(l)} \in L_{G}^{\beta}\left(\Omega_{T}\right), l=1,2$, and $f^{(l)}, g_{i j}^{(l)}$ satisfy $\left(A_{1}\right)$ and $\left(A_{2}\right)$ for some $\beta>1, L>0$. Assume that $\left(Y^{(l)}, Z^{(l)}, K^{(l)}\right) \in S_{G}^{\alpha}(0, T) \times$ $H_{G}^{\alpha}\left(0, T ; \mathbb{R}^{d}\right) \times \mathcal{A}_{G}^{\alpha}(0, T)$, for some $1<\alpha \leq \beta$, are the solutions of equation (4) corresponding to $\xi^{(l)}$, $f^{(l)}$ and $g_{i j}^{(l)}$. Set $\hat{Y}_{t}=Y_{t}^{(1)}-Y_{t}^{(2)}$. Then there exist a constant $C(\alpha)$ depending on $S, G, L$ and $\alpha$ such that

$$
\left|\hat{Y}_{t}\right|^{\alpha} \leq C(\alpha) \hat{\mathbb{E}}_{t}\left[|\hat{\xi}|^{\alpha}+\left(\int_{t}^{T} \hat{h}_{s} d s\right)^{\alpha}\right],
$$

where $\hat{\xi}=\xi^{(1)}-\xi^{(2)}$ and $\hat{h}_{s}=\left|f^{(1)}\left(s, Y_{s}^{(2)}, Z_{s}^{(2)}\right)-f^{(2)}\left(s, Y_{s}^{(2)}, Z_{s}^{(2)}\right)\right|+\sum_{i, j=1}^{d} \mid g_{i j}^{(1)}\left(s, Y_{s}^{(2)}, Z_{s}^{(2)}\right)-$ $g_{i j}^{(2)}\left(s, Y_{s}^{(2)}, Z_{s}^{(2)}\right) \mid$.

Corollary 2.8 (Corollary $\mathbf{5 . 2}$ of [7]) For each $T \leq S$, let $\xi \in L_{G}^{\beta}\left(\Omega_{T}\right)$ and $f, g_{i j}$ satisfy $\left(A_{1}\right)$ and $\left(A_{2}\right)$ for some $\beta>1, L>0$. Assume that $(Y, Z, K) \in S_{G}^{\alpha}(0, T) \times H_{G}^{\alpha}\left(0, T ; \mathbb{R}^{d}\right) \times \mathcal{A}_{G}^{\alpha}(0, T)$, for some $1<\alpha \leq \beta$, is a solution of equation (4) corresponding to $\xi, f$ and $g_{i j}$. Then there exist a constant $C(\alpha)$ depending on $S, G, L$ and $\alpha$ such that

$$
\left|Y_{t}\right|^{\alpha} \leq C(\alpha) \hat{\mathbb{E}}_{t}\left[|\xi|^{\alpha}+\left(\int_{t}^{T} h_{s} d s\right)^{\alpha}\right]
$$

where $h_{s}=|f(s, 0,0)|+\sum_{i, j=1}^{d}\left|g_{i j}(s, 0,0)\right|$.

Proposition 2.9 (Propositions 3.8 of [7]) Let $\xi^{(l)} \in L_{G}^{\beta}\left(\Omega_{T}\right), l=1,2$, and $f^{(l)}, g_{i j}^{(l)}$ satisfy $\left(A_{1}\right)$ and $\left(A_{2}\right)$ for some $\beta>1, L>0$. Assume that $\left(Y^{(l)}, Z^{(l)}, K^{(l)}\right) \in S_{G}^{\alpha}(0, T) \times H_{G}^{\alpha}\left(0, T ; \mathbb{R}^{d}\right) \times \mathcal{A}_{G}^{\alpha}(0, T)$, for some $1<\alpha \leq \beta$, are the solutions of equation (4) corresponding to $\xi^{(l)}, f^{(l)}$ and $g_{i j}^{(l)}$. Set $\hat{Y}_{t}=Y_{t}^{(1)}-Y_{t}^{(2)}, \hat{Z}_{t}=Z_{t}^{(1)}-Z_{t}^{(2)}$. Then there exist a constant $C(\alpha)$ depending on $T, G, L$ and $\alpha$ such that

$$
\hat{\mathbb{E}}\left[\left(\int_{0}^{T}\left|\hat{Z}_{s}\right|^{2} d s\right)^{\frac{\alpha}{2}}\right] \leq C(\alpha)\left\{\|\hat{Y}\|_{S_{G}^{\alpha}}^{\alpha}+\|\hat{Y}\|_{S_{G}^{\alpha}}^{\frac{\alpha}{2}} \sum_{l=1}^{2}\left[\left\|Y^{(l)}\right\|_{S_{G}^{\alpha}}^{\frac{\alpha}{2}}+\left\|\int_{0}^{T} h_{s}^{(l)} d s\right\|_{L_{G}^{\alpha}}^{\frac{\alpha}{2}}\right]\right\},
$$

where $h_{s}^{(l)}=\left|f^{(l)}(s, 0,0)\right|+\sum_{i, j=1}^{d}\left|g_{i j}^{(l)}(s, 0,0)\right|$.

\section{Well-posedness of multi-dimensional G-BSDEs}

In this paper, we shall consider the following type of $n$-dimensional $G$-BSDE on the interval $[0, T]$ :

$$
Y_{t}^{l}=\xi^{l}+\int_{t}^{T} f^{l}\left(s, Y_{s}, Z_{s}^{l}\right) d s+\int_{t}^{T} g_{i j}^{l}\left(s, Y_{s}, Z_{s}^{l}\right) d\left\langle B^{i}, B^{j}\right\rangle_{s}-\int_{t}^{T} Z_{s}^{l} d B_{s}-\left(K_{T}^{l}-K_{t}^{l}\right), 1 \leq l \leq n,
$$

where

$$
f^{l}\left(t, \omega, y, z^{l}\right), g_{i j}^{l}\left(t, \omega, y, z^{l}\right):[0, T] \times \Omega_{T} \times \mathbb{R}^{n} \times \mathbb{R}^{d} \rightarrow \mathbb{R}, \quad \forall 1 \leq l \leq n,
$$

satisfy: 
$\left(H_{1}\right)$ there is some constant $\beta>1$ such that for each $y, z, f^{l}\left(\cdot, \cdot, y, z^{l}\right), g_{i j}^{l}\left(\cdot, \cdot, y, z^{l}\right) \in M_{G}^{\beta}(0, T)$,

$\left(H_{2}\right)$ there exists some $L>0$ such that, for each $y_{1}, y_{2} \in \mathbb{R}^{n}, z_{1}^{l}, z_{2}^{l} \in \mathbb{R}^{d}$,

$$
\left|f^{l}\left(t, y_{1}, z_{1}^{l}\right)-f^{l}\left(t, y_{2}, z_{2}^{l}\right)\right|+\sum_{i, j=1}^{d}\left|g_{i j}^{l}\left(t, y_{1}, z_{1}^{l}\right)-g_{i j}^{l}\left(t, y_{2}, z_{2}^{l}\right)\right| \leq L\left(\left|y_{1}-y_{2}\right|+\left|z_{1}^{l}-z_{2}^{l}\right|\right) .
$$

In the sequel we denote by $M_{G}^{p}\left(a, b ; \mathbb{R}^{n}\right), S_{G}^{\alpha}\left(a, b ; \mathbb{R}^{n}\right), H_{G}^{\alpha}\left(a, b ; \mathbb{R}^{n \times d}\right)$ and $\mathcal{A}_{G}^{\alpha}\left(a, b ; \mathbb{R}^{n}\right)$ the corresponding spaces for the stochastic processes having time indexes on $[a, b]$.

\subsection{Existence and Uniqueness}

We first study the local solution to $G$-BSDE (8). Indeed, we have

Theorem 3.1 Assume that $\left(H_{1}\right)-\left(H_{2}\right)$ hold for some $\beta>1$. Then there exists a constant $0<$ $\delta \leq T$ depending only on $T, G, n, \beta$ and $L$ such that for any $h \in(0, \delta], t \in[0, T-h]$ and given $\zeta \in L_{G}^{\beta}\left(\Omega_{t+h} ; \mathbb{R}^{n}\right)$, the $G-B S D E$ on the interval $[t, t+h]$

$$
\begin{aligned}
Y_{s}^{l}= & \zeta^{l}+\int_{s}^{t+h} f^{l}\left(r, Y_{r}, Z_{r}^{l}\right) d r+\int_{s}^{t+h} g_{i j}^{l}\left(r, Y_{r}, Z_{r}^{l}\right) d\left\langle B^{i}, B^{j}\right\rangle_{r}-\int_{s}^{t+h} Z_{r}^{l} d B_{r}-\left(K_{t+h}^{l}-K_{s}^{l}\right), \\
& 1 \leq l \leq n,
\end{aligned}
$$

admits a unique solution $(Y, Z, K) \in S_{G}^{\alpha}\left(t, t+h ; \mathbb{R}^{n}\right) \times H_{G}^{\alpha}\left(t, t+h ; \mathbb{R}^{n \times d}\right) \times \mathcal{A}_{G}^{\alpha}\left(t, t+h ; \mathbb{R}^{n}\right)$ for each $1<\alpha<\beta$. Moreover, $Y \in M_{G}^{\beta}\left(t, t+h ; \mathbb{R}^{n}\right)$.

In order to prove Theorem 3.1, we consider the following $G$-BSDE on the interval $[t, t+h]$ :

$$
\begin{aligned}
Y_{s}^{U, l}= & \zeta^{l}+\int_{s}^{t+h} f^{l, U}\left(r, Y_{r}^{U, l}, Z_{r}^{U, l}\right) d r+\int_{s}^{t+h} g_{i j}^{l, U}\left(r, Y_{r}^{U, l}, Z_{r}^{U, l}\right) d\left\langle B^{i}, B^{j}\right\rangle_{r}-\int_{s}^{t+h} Z_{r}^{U, l} d B_{r} \\
& -\left(K_{t+h}^{U, l}-K_{s}^{U, l}\right), \quad 1 \leq l \leq n,
\end{aligned}
$$

where $U \in M_{G}^{\beta}\left(t, t+h ; \mathbb{R}^{n}\right), \zeta \in L_{G}^{\beta}\left(\Omega_{t+h} ; \mathbb{R}^{n}\right), h \in[0, T-t]$ and

$\psi^{l, U}\left(t, y^{l}, z^{l}\right)=\psi^{l}\left(t, U_{t}^{1}, \cdots, U_{t}^{l-1}, y^{l}, U_{t}^{l+1}, \cdots, U_{t}^{n}, z^{l}\right):[0, T] \times \Omega_{T} \times \mathbb{R} \times \mathbb{R}^{d} \rightarrow \mathbb{R}, \quad$ for $\psi=f, g_{i j}$.

Denote $X^{U}=\left(X^{U, 1}, \cdots, X^{U, n}\right)$ for $X=Y, Z, K$. Then

Lemma 3.2 Suppose assumptions $\left(H_{1}\right)-\left(H_{2}\right)$ hold for some $\beta>1$. Then, for any $1<\alpha<\beta$, the $G$ $B S D E$ (10) has a unique solution $\left(Y^{U}, Z^{U}, K^{U}\right)$ in $S_{G}^{\alpha}\left(t, t+h ; \mathbb{R}^{n}\right) \times H_{G}^{\alpha}\left(t, t+h ; \mathbb{R}^{n \times d}\right) \times \mathcal{A}_{G}^{\alpha}\left(t, t+h ; \mathbb{R}^{n}\right)$. Moreover, $Y^{U} \in M_{G}^{\beta}\left(t, t+h ; \mathbb{R}^{n}\right)$.

Proof. For each $l \geq 1$, with the help of assumptions $\left(H_{1}\right),\left(H_{2}\right)$ and Lemma 3.3 below, we have $f^{l, U}\left(s, y^{l}, z^{l}\right), g_{i j}^{l, U}\left(s, y^{l}, z^{l}\right) \in M_{G}^{\beta}(t, t+h)$ for each $y^{l} \in \mathbb{R}, z^{l} \in \mathbb{R}^{d}$. Then applying Theorem [2.5 to components of $G$-BSDE (10) yields the existence and uniqueness result of solutions to (10).

Now we shall prove the second part. Let $1 \leq l \leq n$ be given. By Corollary 2.8 we can find some constant $C^{\star}$ depending on $T, G, \beta$ and $L$ such that

$$
\left|Y_{s}^{U, l}\right|^{\beta} \leq C^{\star} \hat{\mathbb{E}}_{s}\left[\left|\zeta^{l}\right|^{\beta}+\left(\int_{s}^{t+h}\left|h_{r}^{l, 0}\right| d r\right)^{\beta}\right]:=\rho_{s}, \forall s \in[t, t+h],
$$


where $h_{s}^{l, 0}=\left|f^{l, U}(s, 0,0)\right|+\left|g_{i j}^{l, U}(s, 0,0)\right|$. Note that $\eta \in L_{G}^{1}\left(\Omega_{t+h}\right)$ implies $\hat{\mathbb{E}}_{s}[|\eta|] \in M_{G}^{1}(t, t+h)$ from a standard approximation argument. Then we have $\rho_{s} \in M_{G}^{1}(t, t+h)$. Now recalling Theorem 4.7 in 11, we conclude that

$$
\lim _{N \rightarrow \infty} \hat{\mathbb{E}}\left[\int_{t}^{t+h}\left|Y_{s}^{U, l}\right|^{\beta} \mid \mathbf{1}_{\left\{\left|Y_{s}^{l}\right|^{\beta} \geq N\right\}}\right] \leq \lim _{N \rightarrow \infty} \hat{\mathbb{E}}\left[\int_{t}^{t+h} \rho_{s} \mathbf{1}_{\left\{\rho_{s} \geq N\right\}}\right]=0,
$$

which, together with the fact that $\left(Y_{s}^{U, l}\right)_{t \leq s \leq t+h} \in S_{G}^{\alpha}(t, t+h) \subset M_{G}^{\alpha}(t, t+h)$, indicates that $Y^{U, l} \in$ $M_{G}^{\beta}(t, t+h)$. The proof is complete.

Lemma 3.3 Suppose $p \geq 1$. Given a function $h:[t, t+h] \times \Omega_{T} \times \mathbb{R}^{m} \rightarrow \mathbb{R}$ satisfying $h(\cdot, \cdot, x) \in$ $M_{G}^{p}(t, t+h)$ for each $x \in \mathbb{R}^{m}$. Assume that $h$ is Lipschitz continuous in $x$ with some coefficient $L$, uniformly in $(t, \omega)$. Then for any $X \in M_{G}^{p}\left(t, t+h ; \mathbb{R}^{m}\right)$, we have $\left(h\left(s, X_{s}\right)\right)_{t \leq s \leq t+h} \in M_{G}^{p}(t, t+h)$.

Proof. According to the partition of unit theorem, for each integer $k \geq 1$, we can find a sequence of continuous functions $\left\{\phi_{i}^{k}\right\}_{i=1}^{\infty}$ such that the diameter of support $\lambda\left(\operatorname{supp}\left(\phi_{i}^{k}\right)\right) \leq 1 / k$ and $0 \leq \phi_{i}^{k} \leq 1$. Moreover, for any integer $N \geq 1$, there exists a constant $k_{N}$ such that

$$
I_{B_{N}(0)}(x) \leq \sum_{i=1}^{k_{N}} \phi_{i}^{k}(x) \leq 1,
$$

where $B_{N}(0)$ denotes the open ball centered at 0 with radius $N$. Choose $x_{i}^{k} \in \mathbb{R}^{m}$ such that $\phi_{i}^{k}\left(x_{i}^{k}\right)>0$. By Theorem 4.7 in [11], we have $\left(\phi_{i}^{k}\left(X_{s}\right) h\left(s, x_{i}^{k}\right)\right)_{t \leq s \leq t+h} \in M_{G}^{p}(t, t+h)$, which implies that

$$
\left(\sum_{i=1}^{k_{N}} \phi_{i}^{k}\left(X_{s}\right) h\left(s, x_{i}^{k}\right)\right)_{t \leq s \leq t+h} \in M_{G}^{p}(t, t+h) .
$$

Note that

$$
\begin{aligned}
\left|\sum_{i=1}^{k_{N}} \phi_{i}^{k}\left(X_{s}\right) h\left(s, x_{i}^{k}\right)-h\left(s, X_{s}\right)\right| & \leq \sum_{i=1}^{k_{N}} \phi_{i}^{k}\left(X_{s}\right)\left|h\left(s, x_{i}^{k}\right)-h\left(s, X_{s}\right)\right|+\left(1-\sum_{i=1}^{k_{N}} \phi_{i}^{k}\left(X_{s}\right)\right) h\left(s, X_{s}\right) \\
& \leq L \sum_{i=1}^{k_{N}} \phi_{i}^{k}\left(X_{s}\right)\left|X_{s}-x_{i}^{k}\right|+I_{\left\{\left(B_{N}(0)\right)^{c}\right\}}\left(X_{s}\right) h\left(s, X_{s}\right) \\
& \leq L \sum_{i=1}^{k_{N}} \phi_{i}^{k}\left(X_{s}\right) \frac{1}{k}+\left(L\left|X_{s}\right|+h(s, 0)\right) I_{\left\{\left|X_{s}\right| \geq N\right\}} \\
& \leq L \frac{1}{k}+\left(L\left|X_{s}\right|+h(s, 0)\right) I_{\left\{\left|X_{s}\right| \geq N\right\}} .
\end{aligned}
$$

Consequently, applying Theorem 4.7 in [1] again, we get that

$$
\hat{\mathbb{E}}\left[\int_{t}^{t+h}\left|\sum_{i=1}^{k_{N}} \phi_{i}^{k}\left(X_{s}\right) h\left(s, x_{i}^{k}\right)-h\left(s, X_{s}\right)\right|^{p} d s\right] \rightarrow 0, \quad \text { as } k, N \rightarrow \infty,
$$

which implies the desired result.

In the view of Lemma 3.2, we can define the solution map $\Gamma: U \rightarrow \Gamma(U)$ from $M_{G}^{\beta}\left(t, t+h ; \mathbb{R}^{n}\right)$ to $M_{G}^{\beta}\left(t, t+h ; \mathbb{R}^{n}\right)$ by

$$
\Gamma(U):=Y^{U}, \quad \forall U \in M_{G}^{\beta}\left(t, t+h ; \mathbb{R}^{n}\right) .
$$

The following lemma shows that the solution map $\Gamma$ is a contraction whenever $h$ is small enough. 
Lemma 3.4 Assume $\left(H_{1}\right)-\left(H_{2}\right)$ hold for some $\beta>1$. Then there is a constant $\delta>0$ depending only on $T, G, n, \beta$ and $L$ such that for any $h \in(0, \delta]$, we have

$$
\left\|Y^{U}-Y^{\bar{U}}\right\|_{M_{G}^{\beta}\left(t, t+h ; \mathbb{R}^{n}\right)} \leq \frac{1}{2}\|U-\bar{U}\|_{M_{G}^{\beta}\left(t, t+h ; \mathbb{R}^{n}\right)}, \quad \forall U, \bar{U} \in M_{G}^{\beta}\left(t, t+h ; \mathbb{R}^{n}\right) .
$$

Proof. For each $1 \leq l \leq n$, recall that $Y^{U, l}$ and $Y^{\bar{U}, l}$ are the $l$-th components of $Y^{U}$ and $Y^{\bar{U}}$, respectively. Applying Proposition 2.7 to $\left(Y^{U, l}-Y^{\bar{U}, l}\right)$ yields that

$$
\left|Y_{s}^{U, l}-Y_{s}^{\bar{U}, l}\right|^{\beta} \leq C^{\star} \hat{\mathbb{E}}_{s}\left[\left(\int_{s}^{t+h} \hat{h}_{r} d r\right)^{\beta}\right], \quad \forall s \in[t, t+h],
$$

where

$$
\hat{h}_{s}=\left|f^{l, U}\left(s, Y_{s}^{\bar{U}, l}, Z_{s}^{\bar{U}, l}\right)-f^{l, \bar{U}}\left(s, Y_{s}^{\bar{U}, l}, Z_{s}^{\bar{U}, l}\right)\right|+\sum_{i, j=1}^{d}\left|g_{i j}^{l, U}\left(s, Y_{s}^{\bar{U}, l}, Z_{s}^{\bar{U}, l}\right)-g_{i j}^{l, \bar{U}}\left(s, Y_{s}^{\bar{U}, l}, Z_{s}^{\bar{U}, l}\right)\right|
$$

and $C^{\star}$ is a constant depending on $T, G, \beta$ and $L$. From assumption $\left(H_{2}\right)$ and the Hölder's inequality, we then get

$$
\hat{\mathbb{E}}\left[\left|Y_{s}^{U, l}-Y_{s}^{\bar{U}, l}\right|^{\beta}\right] \leq C^{\star} L^{\beta} h^{\beta-1} \hat{\mathbb{E}}\left[\int_{t}^{t+h}\left|U_{r}-\bar{U}_{r}\right|^{\beta} d r\right], \quad \forall s \in[t, t+h] .
$$

Note that for each $y_{1}, y_{2} \in \mathbb{R}^{n}$,

$$
\left|y_{1}-y_{2}\right|^{\beta}=\left(\sum_{l=1}^{n}\left|y_{1}^{l}-y_{2}^{l}\right|^{2}\right)^{\frac{\beta}{2}} \leq n^{\max \left(\frac{\beta}{2}-1,0\right)}\left(\sum_{l=1}^{n}\left|y_{1}^{l}-y_{2}^{l}\right|^{\beta}\right) .
$$

Then we derive that for $s \in[t, t+h]$,

$$
\hat{\mathbb{E}}\left[\left|Y_{s}^{U}-Y_{s}^{\bar{U}}\right|^{\beta}\right] \leq n^{\max \left(\frac{\beta}{2}-1,0\right)} \sum_{l=1}^{n} \hat{\mathbb{E}}\left[\left|Y_{s}^{U, l}-Y_{s}^{\bar{U}, l}\right|^{\beta}\right] \leq n^{\max \left(\frac{\beta}{2}, 1\right)} C^{\star} L^{\beta} h^{\beta-1} \hat{\mathbb{E}}\left[\int_{t}^{t+h}\left|U_{r}-\bar{U}_{r}\right|^{\beta} d r\right] .
$$

Consequently,

$$
\left\|Y^{U}-Y^{\bar{U}}\right\|_{M_{G}^{\beta}\left(t, t+h ; \mathbb{R}^{n}\right)} \leq\left|\int_{t}^{t+h} \hat{\mathbb{E}}\left[\left|Y_{s}^{U}-Y_{s}^{\bar{U}}\right|^{\beta}\right] d s\right|^{\frac{1}{\beta}} \leq n^{\max \left(\frac{1}{2}, \frac{1}{\beta}\right)}\left|C^{\star}\right|^{\frac{1}{\beta}} L h\|U-\bar{U}\|_{M_{G}^{\beta}\left(t, t+h ; \mathbb{R}^{n}\right)^{.}} .
$$

By setting

$$
\delta:=\min \left(\frac{1}{2 n^{\max \left(\frac{1}{2}, \frac{1}{\beta}\right)}\left|C^{\star}\right|^{\frac{1}{\beta}} L}, T\right),
$$

we will have, for each $h \in(0, \delta]$,

$$
\left\|Y^{U}-Y^{\bar{U}}\right\|_{M_{G}^{\beta}\left(t, t+h ; \mathbb{R}^{n}\right)} \leq \frac{1}{2}\|U-\bar{U}\|_{M_{G}^{\beta}\left(t, t+h ; \mathbb{R}^{n}\right)},
$$

as desired.

Now we are going to use the method of Picard iteration to obtain the existence and uniqueness of local solutions as following, which is also crucial for the establishment of comparison theorem in the sequel.

The proof of Theorem 3.1, For symbol simplicity, we assume that $g_{i j} \equiv 0$, and the proof still holds for the general case. In the following, unless otherwise stated, $C$ will always denote a generic constant which may change from line to line. The proof shall be divided into three steps 
1 The convergence of $Y$. We take $\delta$ as in Lemma 3.4 For any given $0 \leq h \leq \delta$, let $Y_{s}^{(0)} \equiv 0$ and $Y_{s}^{(i+1)}:=Y_{s}^{Y^{(i)}}$ be iterated defined by the following multi-dimensional $G$-BSDE on $[t, t+h]$ :

$Y_{s}^{(i+1), l}=\zeta^{l}+\int_{s}^{t+h} f^{l, Y^{(i)}}\left(r, Y_{r}^{(i+1), l}, Z_{r}^{(i+1), l}\right) d r-\int_{s}^{t+h} Z_{r}^{(i+1), l} d B_{r}-\left(K_{t+h}^{(i+1), l}-K_{s}^{(i+1), l}\right), 1 \leq l \leq n$.

Recalling Lemma 3.4, we can get that for each $i$,

$$
\left\|Y^{(i+1)}-Y^{(i)}\right\|_{M_{G}^{\beta}\left(t, t+h ; \mathbb{R}^{n}\right)} \leq \frac{1}{2}\left\|Y^{(i)}-Y^{(i-1)}\right\|_{M_{G}^{\beta}\left(t, t+h ; \mathbb{R}^{n}\right)} \leq \cdots \leq \frac{1}{2^{i}}\left\|Y^{(1)}-Y^{(0)}\right\|_{M_{G}^{\beta}\left(t, t+h ; \mathbb{R}^{n}\right)},
$$

from which, we derive that $Y^{(i)}$ is a Cauchy sequence in $M_{G}^{\beta}\left(t, t+h ; \mathbb{R}^{n}\right)$.

For each $1 \leq l \leq n$, applying the estimate (5) in Proposition 2.7 to $Y^{(i+1), l}-Y^{(j+1), l}$, we get

$$
\left|Y_{s}^{(i+1), l}-Y_{s}^{(j+1), l}\right|^{\alpha} \leq C \hat{\mathbb{E}}_{s}\left[\left(\int_{s}^{t+h} \hat{h}_{r}^{l} d r\right)^{\alpha}\right] \leq C \hat{\mathbb{E}}_{s}\left[\left(\int_{t}^{t+h}\left|Y_{r}^{(i)}-Y_{r}^{(j)}\right| d r\right)^{\alpha}\right]
$$

where $\hat{h}_{s}^{l}=\left|f^{l, Y^{(i)}}\left(s, Y_{s}^{(i+1), l}, Z_{s}^{(i+1), l}\right)-f^{l, Y^{(j)}}\left(s, Y_{s}^{(i+1), l}, Z_{s}^{(i+1), l}\right)\right|$. Then it follows from Theorem 2.2 that

$$
\begin{aligned}
\hat{\mathbb{E}}\left[\sup _{t \leq s \leq t+h}\left|Y_{s}^{(i+1), l}-Y_{s}^{(j+1), l}\right|^{\alpha}\right] & \leq C \hat{\mathbb{E}}\left[\sup _{t \leq s \leq t+h} \hat{\mathbb{E}}_{s}\left[\left(\int_{t}^{t+h}\left|Y_{r}^{(i)}-Y_{r}^{(j)}\right| d r\right)^{\alpha}\right]\right] \\
& \left.\leq C \hat{\mathbb{E}}\left[\left(\int_{t}^{t+h}\left|Y_{r}^{(i)}-Y_{r}^{(j)}\right| d r\right)^{\beta}\right]\right)^{\frac{\alpha}{\beta}} \\
& \left.\leq C \hat{\mathbb{E}}\left[\int_{t}^{t+h}\left|Y_{r}^{(i)}-Y_{r}^{(j)}\right|^{\beta} d r\right]\right)^{\frac{\alpha}{\beta}} .
\end{aligned}
$$

Consequently, we obtain that $Y^{(i)}$ is a Cauchy sequence in $S_{G}^{\alpha}\left(t, t+h ; \mathbb{R}^{n}\right)$. In particular, there exists some process $Y$ such that $Y^{(i)}$ converges to $Y$ in $S_{G}^{\alpha}\left(t, t+h ; \mathbb{R}^{n}\right)$. Moreover, we have

$$
\left\|Y^{(i)}\right\|_{S_{G}^{\alpha}\left(t, t+h ; \mathbb{R}^{n}\right)} \leq C \text {, uniformly for all } i .
$$

2 The existence. Now applying the estimate (7) in Proposition 2.9 to $Z^{(i+1), l}-Z^{(j+1), l}$, we derive that

$$
\begin{aligned}
& \hat{\mathbb{E}}\left[\left(\int_{t}^{t+h}\left|Z_{s}^{(i+1), l}-Z_{s}^{(j+1), l}\right|^{2} d s\right)^{\frac{\alpha}{2}}\right] \\
& \leq C\left\{\left\|Y^{(i+1), l}-Y^{(j+1), l}\right\|_{S_{G}^{\alpha}\left(t, t+h ; \mathbb{R}^{n}\right)}^{\alpha}+\left\|Y^{(i+1), l}-Y^{(j+1), l}\right\|_{S_{G}^{\alpha}\left(t, t+h ; \mathbb{R}^{n}\right)}^{\frac{\alpha}{2}}\left[\left.\left\|Y^{(i+1), l}\right\|\right|_{S_{G}^{\alpha}} ^{\frac{\alpha}{2}}\left(t, t+h ; \mathbb{R}^{n}\right)\right.\right. \\
& \left.\left.+\left\|Y^{(j+1), l}\right\|_{S_{G}^{\alpha}\left(t, t+h ; \mathbb{R}^{n}\right)}^{\frac{\alpha}{2}}+\left\|\int_{t}^{t+h}\left|f^{l, Y^{(i)}}(s, 0,0)\right| d s\right\|_{L_{G}^{\alpha}\left(\Omega_{t+h} ; \mathbb{R}^{n}\right)}^{\frac{\alpha}{2}}+\left\|\int_{t}^{t+h}\left|f^{l, Y^{(j)}}(s, 0,0)\right| d s\right\|_{L_{G}^{\alpha}\left(\Omega_{t+h} ; \mathbb{R}^{n}\right)}^{\frac{\alpha}{2}}\right]\right\} \\
& \leq C\left\{\left\|Y^{(i+1), l}-Y^{(j+1), l}\right\|_{S_{G}^{\alpha}\left(t, t+h ; \mathbb{R}^{n}\right)}^{\alpha}+\left\|Y^{(i+1), l}-Y^{(j+1), l}\right\|_{S_{G}^{\alpha}\left(t, t+h ; \mathbb{R}^{n}\right)}^{\frac{\alpha}{2}}\right\},
\end{aligned}
$$

where we have used the assumptions $\left(H_{1}\right)-\left(H_{2}\right)$ and the estimate (13) in the last inequality. Therefore, it follows from Step 1 that $Z^{(i)}$ is a Cauchy sequence in $H_{G}^{\alpha}\left(t, t+h ; \mathbb{R}^{n \times d}\right)$, and thus converges to some $Z \in H_{G}^{\alpha}\left(t, t+h ; \mathbb{R}^{n \times d}\right)$.

For each $1 \leq l \leq n$, we define

$$
K_{s}^{l}=Y_{s}^{l}+\int_{t}^{s} f^{l}\left(r, Y_{r}, Z_{r}^{l}\right) d r-\int_{t}^{s} Z_{r}^{l} d B_{r}-Y_{t}^{l}, \forall t \leq s \leq t+h .
$$


Then it is easy to check that $K_{s}^{(i)}$ converges to $K_{s}$ in $L_{G}^{\alpha}\left(\Omega_{s} ; \mathbb{R}^{n}\right)$ and $K \in \mathcal{A}_{G}^{\alpha}\left(t, t+h ; \mathbb{R}^{n}\right)$. This prove the existence.

3 The uniqueness. Let $\left(Y^{\prime}, Z^{\prime}, K^{\prime}\right)$ be another solution of equation (9). Note that $Y, Y^{\prime} \in$ $M_{G}^{\beta}\left(t, t+h ; \mathbb{R}^{n}\right)$ are both fixed points of the mapping $\Gamma$, then Lemma 3.4 implies that $Y=Y^{\prime}$. Thus $(Y, Z, K)$ and $\left(Y^{\prime}, Z^{\prime}, K^{\prime}\right)$ can be viewed as the solution to the following $G$-BSDE:

$$
\bar{Y}_{s}^{l}=\zeta^{l}+\int_{s}^{t+h} f^{l}\left(r, Y_{r}, \bar{Z}_{r}^{l}\right) d r-\int_{s}^{t+h} \bar{Z}_{r}^{l} d B_{r}-\left(\bar{K}_{t+h}^{l}-\bar{K}_{s}^{l}\right), t \leq s \leq t+h, 1 \leq l \leq n .
$$

Therefore, applying Theorem 2.5 to components of the above equation yields that $(Z, K)=\left(Z^{\prime}, K^{\prime}\right)$. The proof is complete.

Now we are in a position to state the well-posedness for $G$-BSDE (8) on the whole interval $[0, T]$.

Theorem 3.5 Suppose $\left(H_{1}\right)-\left(H_{2}\right)$ are satisfied for some $\beta>1$. Then for any $1<\alpha<\beta$, the $G$ $B S D E$ (8) has a unique solution $(Y, Z, K) \in S_{G}^{\alpha}\left(0, T ; \mathbb{R}^{n}\right) \times H_{G}^{\alpha}\left(0, T ; \mathbb{R}^{n \times d}\right) \times \mathcal{A}_{G}^{\alpha}\left(0, T ; \mathbb{R}^{n}\right)$. Moreover, $Y \in M_{G}^{\beta}\left(0, T ; \mathbb{R}^{n}\right)$.

Proof. Choose integer $m$ large enough such that $m \delta \geq T$, where $\delta$ is given by Theorem 3.1 Taking $h=\frac{T}{m}$ and applying Theorem 3.1] we deduce that the $G$-BSDE (8) has a unique solution $\left(Y^{(m)}, Z^{(m)}, K^{(m)}\right) \in S_{G}^{\alpha}\left(T-h, T ; \mathbb{R}^{n}\right) \times H_{G}^{\alpha}\left(T-h, T ; \mathbb{R}^{n \times d}\right) \times \mathcal{A}_{G}^{\alpha}\left(T-h, T ; \mathbb{R}^{n}\right)$ on the time interval $[T-h, T]$. Next we take $T-h$ as the terminal time and $Y_{T-h}^{(m)}$ as the terminal condition. Applying Theorem 3.1 again, we deduce that the $G$-BSDE (8) admits a unique solution $\left(Y^{(m-1)}, Z^{(m-1)}, K^{(m-1)}\right) \in S_{G}^{\alpha}\left(T-2 h, T-h ; \mathbb{R}^{n}\right) \times H_{G}^{\alpha}\left(T-2 h, T-h ; \mathbb{R}^{n \times d}\right) \times \mathcal{A}_{G}^{\alpha}\left(T-2 h, T-h ; \mathbb{R}^{n}\right)$ on the time interval $[T-2 h, T-h]$. Repeating this procedure, we obtain a solution sequence $\left(Y^{(i)}, Z^{(i)}, K^{(i)}\right)_{i \leq m}$. Set

$$
Y_{t}=\sum_{i=1}^{m} Y_{t}^{(i)} I_{[(i-1) h, i h)}(t)+Y_{T}^{(m)} I_{\{T\}}(t), Z_{t}=\sum_{i=1}^{m} Z_{t}^{(i)} I_{[(i-1) h, i h)}(t)+Z_{T}^{(m)} I_{\{T\}}(t)
$$

and

$$
K_{t}=K_{t}^{(i)}+\sum_{j=1}^{i-1} K_{j h}^{(j)} \text {, for } t \in[(i-1) h, i h) \text { and } K_{T}=K_{T}^{(m)}+\sum_{j=1}^{m-1} K_{j h}^{(j)} .
$$

It is easy to check that $(Y, Z, K) \in S_{G}^{\alpha}\left(0, T ; \mathbb{R}^{n}\right) \times H_{G}^{\alpha}\left(0, T ; \mathbb{R}^{n \times d}\right) \times \mathcal{A}_{G}^{\alpha}\left(0, T ; \mathbb{R}^{n}\right)$ is a solution to $G$-BSDE (8) and $Y \in M_{G}^{\beta}\left(0, T ; \mathbb{R}^{n}\right)$. Thus we get the existence. The uniqueness follows from the one on each small interval. The proof is complete.

We conclude this subsection with the comparison theorem for multi-dimensional $G$-BSDEs (8). Consider the following two $G$-BSDEs on the interval $[0, T]$ :

$$
\begin{aligned}
& Y_{t}^{l}=\xi^{l}+\int_{t}^{T} f^{l}\left(s, Y_{s}, Z_{s}^{l}\right) d s+\int_{t}^{T} g_{i j}^{l}\left(s, Y_{s}, Z_{s}^{l}\right) d\left\langle B^{i}, B^{j}\right\rangle_{s}-\int_{t}^{T} Z_{s}^{l} d B_{s}-\left(K_{T}^{l}-K_{t}^{l}\right), \quad 1 \leq l \leq n, \\
& \bar{Y}_{t}^{l}=\bar{\xi}^{l}+\int_{t}^{T} \bar{f}^{l}\left(s, \bar{Y}_{s}, \bar{Z}_{s}^{l}\right) d s+\int_{t}^{T} \bar{g}_{i j}^{l}\left(s, \bar{Y}_{s}, \bar{Z}_{s}^{l}\right) d\left\langle B^{i}, B^{j}\right\rangle_{s}-\int_{t}^{T} \bar{Z}_{s}^{l} d B_{s}-\left(\bar{K}_{T}^{l}-\bar{K}_{t}^{l}\right), \quad 1 \leq l \leq n .
\end{aligned}
$$

Then we have

Theorem 3.6 Suppose that $f^{l}\left(t, y, z^{l}\right), \bar{f}^{l}\left(t, \bar{y}, z^{l}\right), g_{i j}^{l}\left(t, y, z^{l}\right), \bar{g}_{i j}^{l}\left(t, \bar{y}, z^{l}\right)$ satisfy $\left(H_{1}\right)-\left(H_{2}\right)$ and $\xi, \bar{\xi} \in$ $L_{G}^{\beta}\left(\Omega_{T}\right)$ for some $\beta>1$. Assume the following conditions hold: 
(i) for any $1 \leq l \leq n$, and for each $t \in[0, T], z^{l} \in \mathbb{R}^{d}$ and $y, \bar{y} \in \mathbb{R}^{n}$ satisfying $y^{j} \geq \bar{y}^{j}$ for $j \neq l$ and $y^{l}=\bar{y}^{l}$, it holds that $f^{l}\left(t, y, z^{l}\right) \geq \bar{f}^{l}\left(t, \bar{y}, z^{l}\right),\left[g_{i j}^{l}\left(t, y, z^{l}\right)\right]_{i, j=1}^{d} \geq\left[\bar{g}_{i j}^{l}\left(t, \bar{y}, z^{l}\right)\right]_{i, j=1}^{d} ;$

(ii) $\xi \geq \bar{\xi}$.

Then we have $Y_{t} \geq \bar{Y}_{t}$ for each $t \in[0, T]$.

Proof. For symbol simplicity, we assume $g_{i j}^{l} \equiv \bar{g}_{i j}^{l} \equiv 0$, and the proof for the general case is just similar. According to the proof of Theorem [3.5, it suffices to prove the theorem on the interval $[T-h, T]$, since the comparison principle on $[0, T]$ can be obtained backwardly by iteration. In the following we will prove the conclusion based on the Picard iteration obtained in the proof of Theorem 3.1

For each $i \in \mathbb{N}$ and $1 \leq l \leq n$, let $Y^{(i), l}$ be iterated defined by $Y_{t}^{(0)} \equiv 0$ and

$$
Y_{t}^{(i), l}=\xi^{l}+\int_{t}^{T} f^{l, Y^{(i-1)}}\left(s, Y_{s}^{(i), l}, Z_{s}^{(i), l}\right) d s-\int_{t}^{T} Z_{s}^{(i), l} d B_{s}-\left(K_{T}^{(i), l}-K_{t}^{(i), l}\right), T-h \leq t \leq T .
$$

Similarly, let $\bar{Y}^{(i), l}$ be iterated defined by $\bar{Y}_{t}^{(0)} \equiv 0$ and

$$
\bar{Y}_{t}^{(i), l}=\bar{\xi}^{l}+\int_{t}^{T} \bar{f}^{l, \bar{Y}^{(i-1)}}\left(s, \bar{Y}_{s}^{(i), l}, \bar{Z}_{s}^{(i), l}\right) d s-\int_{t}^{T} \bar{Z}_{s}^{(i), l} d B_{s}-\left(\bar{K}_{T}^{(i), l}-\bar{K}_{t}^{(i), l}\right), T-h \leq t \leq T .
$$

For $i=0$, from conditions (i) and (ii), we can apply the comparison theorem of one-dimensional $G$-BSDEs (Theorem 2.6) to the components of the above two equations and get

$$
Y_{t}^{(1)} \geq \bar{Y}_{t}^{(1)}, \quad T-h \leq t \leq T .
$$

Repeating the above procedure, we have

$$
Y_{t}^{(i)} \geq \bar{Y}_{t}^{(i)}, \quad T-h \leq t \leq T .
$$

Letting $i \rightarrow \infty$, according to the proof of Theorem 3.1 we obtain finally

$$
Y_{t} \geq \bar{Y}_{t}, \quad T-h \leq t \leq T,
$$

which is the desired result.

Remark 3.7 In the routine method of Picard iteration, the iteration equation (10) takes the form

$$
\begin{aligned}
Y_{s}^{U, l}= & \zeta^{l}+\int_{s}^{t+h} f^{l}\left(r, U_{r}, Z_{r}^{U, l}\right) d r+\int_{s}^{t+h} g_{i j}^{l}\left(r, U_{r}, Z_{r}^{U, l}\right) d\left\langle B^{i}, B^{j}\right\rangle_{r}-\int_{s}^{t+h} Z_{r}^{U, l} d B_{r} \\
& -\left(K_{t+h}^{U, l}-K_{s}^{U, l}\right), \quad 1 \leq l \leq n .
\end{aligned}
$$

But this formulation can only, in the above limit argument, obtain the comparison theorem under a assumption stronger than (i) in Theorem 3.6 on generators that

(i') for any $1 \leq l \leq n$, and for each $t \in[0, T], z^{l} \in \mathbb{R}^{d}$ and $y, \bar{y} \in \mathbb{R}^{n}$ such that $y^{j} \geq \bar{y}^{j}$ for each $j$, we have $f^{l}\left(t, y, z^{l}\right) \geq \bar{f}^{l}\left(t, \bar{y}, z^{l}\right),\left[g_{i j}^{l}\left(t, y, z^{l}\right)\right]_{i, j=1}^{d} \geq\left[\bar{g}_{i j}^{l}\left(t, \bar{y}, z^{l}\right)\right]_{i, j=1}^{d}$,

which, when $n=1$, even does not contain the comparison theorem for one-dimensional $G$-BSDEs. The advantage that we adopt a new form of generators as in (10) is that we can get the comparison theorem in a general form similar to the classical case. 


\subsection{Some useful estimates}

In this section, we shall present some estimates for $Y$. For symbol simplicity, we will denote

$$
\phi(t, y, z)=\left(\phi^{1}\left(t, y, z^{1}\right), \phi^{2}\left(t, y, z^{2}\right), \cdots, \phi^{n}\left(t, y, z^{n}\right)\right)^{T}, \quad \text { for } \phi=f, g_{i j} .
$$

Then it holds that

Proposition 3.8 Let ${ }^{\nu} \xi \in L_{G}^{\beta}\left(\Omega_{T} ; \mathbb{R}^{n}\right)$, and ${ }^{\nu} f,{ }^{\nu} g_{i j}$ satisfy $(H 1)$-(H2) for some $\beta>1, \nu=1,2$. Assume that $\left({ }^{\nu} Y,{ }^{\nu} Z,{ }^{\nu} K\right) \in S_{G}^{\alpha}\left(0, T ; \mathbb{R}^{n}\right) \times H_{G}^{\alpha}\left(0, T ; \mathbb{R}^{n \times d}\right) \times \mathcal{A}_{G}^{\alpha}\left(0, T ; \mathbb{R}^{n}\right)$ for some $1<\alpha \leq \beta$, are the solutions of equation (8) corresponding to ${ }^{\nu} \xi,{ }^{\nu} f$ and ${ }^{\nu} g_{i j}$. Set $\hat{Y}_{t}={ }^{1} Y_{t}-{ }^{2} Y_{t}$. Then there exists a constant $C(\alpha, n, T)$ depending only on $T, G, n, L$ and $\alpha$ such that

$$
\left|\hat{Y}_{t}\right|^{\alpha} \leq C(\alpha, n, T) \hat{\mathbb{E}}_{t}\left[|\hat{\xi}|^{\alpha}+\left(\int_{t}^{T} \hat{h}_{s} d s\right)^{\alpha}\right]
$$

where $\hat{\xi}={ }^{1} \xi-{ }^{2} \xi, \hat{h}_{s}=\left|{ }^{1} f\left(s,{ }^{2} Y_{s},{ }^{2} Z_{s}\right)-{ }^{2} f\left(s,{ }^{2} Y_{s},{ }^{2} Z_{s}\right)\right|+\sum_{i, j=1}^{d}\left|{ }^{1} g_{i j}\left(s,{ }^{2} Y_{s},{ }^{2} Z_{s}\right)-{ }^{2} g_{i j}\left(s,{ }^{2} Y_{s},{ }^{2} Z_{s}\right)\right|$.

Proof. For any $1 \leq l \leq n$, applying Proposition 2.7 to $\left({ }^{1} Y^{l}-{ }^{2} Y^{l}\right)$ yields that, for each $s \in[t, T]$,

$$
\left|\hat{Y}_{s}^{l}\right|^{\alpha} \leq C(\alpha) \hat{\mathbb{E}}_{s}\left[\left|\hat{\xi}^{l}\right|^{\alpha}+\left(\int_{s}^{T} k_{r}^{l} d r\right)^{\alpha}\right] \leq C(\alpha) \hat{\mathbb{E}}_{s}\left[|\hat{\xi}|^{\alpha}+\left(\int_{s}^{T} \hat{h}_{r} d r\right)^{\alpha}+\left(\int_{s}^{T}\left|\hat{Y}_{r}\right| d r\right)^{\alpha}\right],
$$

where $k_{r}^{l}=\left|{ }^{1} f^{l}\left(r,{ }^{1} Y_{r},{ }^{2} Z_{r}^{l}\right)-{ }^{2} f^{l}\left(r,{ }^{2} Y_{r},{ }^{2} Z_{r}^{l}\right)\right|+\sum_{i, j=1}^{d}\left|{ }^{1} g_{i j}^{l}\left(r,{ }^{1} Y_{r},{ }^{2} Z_{r}^{l}\right)-{ }^{2} g_{i j}^{l}\left(r,{ }^{2} Y_{r},{ }^{2} Z_{r}^{l}\right)\right|$. Denote by $C(\alpha, n, T)$ a generic constant depending on $T, G, n, L$ and $\alpha$, which may vary from line to line. Summing up (15) over $l$, we conclude that

$$
\left|\hat{Y}_{s}\right|^{\alpha} \leq C(\alpha, n, T)\left(\hat{\mathbb{E}}_{s}\left[|\hat{\xi}|^{\alpha}+\left(\int_{t}^{T} \hat{h}_{r} d r\right)^{\alpha}\right]+\int_{s}^{T} \hat{\mathbb{E}}_{s}\left[\left|\hat{Y}_{r}\right|^{\alpha}\right] d r\right), s \geq t .
$$

Taking conditional expectation $\hat{\mathbb{E}}_{t}$ on both sides, we get

$$
\hat{\mathbb{E}}_{t}\left[\left|\hat{Y}_{s}\right|^{\alpha}\right] \leq C(\alpha, n, T)\left(\hat{\mathbb{E}}_{t}\left[|\hat{\xi}|^{\alpha}+\left(\int_{t}^{T} \hat{h}_{r} d r\right)^{\alpha}\right]+\int_{s}^{T} \hat{\mathbb{E}}_{t}\left[\left|\hat{Y}_{r}\right|^{\alpha}\right] d r\right), s \geq t .
$$

Consequently, from Gronwall's inequality, we obtain that

$$
\hat{\mathbb{E}}_{t}\left[\left|\hat{Y}_{s}\right|^{\alpha}\right] \leq C(\alpha, n, T)\left(\hat{\mathbb{E}}_{t}\left[|\hat{\xi}|^{\alpha}+\left(\int_{t}^{T} \hat{h}_{r} d r\right)^{\alpha}\right]\right), s \geq t
$$

which ends the proof.

Corollary 3.9 Let $\xi \in L_{G}^{\beta}\left(\Omega_{T} ; \mathbb{R}^{n}\right)$ and $f, g_{i j}$ satisfy $(H 1)-(H 2)$ for some $\beta>1$. Assume that $(Y, Z, K) \in S_{G}^{\alpha}\left(0, T ; \mathbb{R}^{n}\right) \times H_{G}^{\alpha}\left(0, T ; \mathbb{R}^{n \times d}\right) \times \mathcal{A}_{G}^{\alpha}\left(0, T ; \mathbb{R}^{n}\right)$ for some $1<\alpha \leq \beta$, is a solution of equation (8) corresponding to $\xi, f$ and $g_{i j}$. Then there exists a constant $C(\alpha, n, T)$ depending only on $T, G, L, n$ and $\alpha$ such that

$$
\left|Y_{t}\right|^{\alpha} \leq C(\alpha, n, T) \hat{\mathbb{E}}_{t}\left[|\xi|^{\alpha}+\left(\int_{t}^{T} h_{s} d s\right)^{\alpha}\right]
$$

where $h_{s}=|f(s, 0,0)|+\sum_{i, j=1}^{d}\left|g_{i j}(s, 0,0)\right|$.

Proof. Taking ${ }^{2} \xi \equiv 0,{ }^{2} f \equiv{ }^{2} g_{i j} \equiv 0$ in Proposition 3.8 and noting that ${ }^{2} Y \equiv 0,{ }^{2} Z \equiv 0,{ }^{2} K \equiv 0$, we get the desired conclusion. 
Proposition 3.10 Let $\xi \in L_{G}^{\beta}\left(\Omega_{T} ; \mathbb{R}^{n}\right)$ and $f, g_{i j}$ satisfy $(H 1)-(H 2)$ for some $\beta>1$. Assume that $(Y, Z, K) \in S_{G}^{\alpha}\left(0, T ; \mathbb{R}^{n}\right) \times H_{G}^{\alpha}\left(0, T ; \mathbb{R}^{n \times d}\right) \times \mathcal{A}_{G}^{\alpha}\left(0, T ; \mathbb{R}^{n}\right)$ for some $1<\alpha<\beta$, is a solution of equation (8) corresponding to $\xi, f$ and $g_{i j}$. Let any $1<\alpha<\alpha^{\prime} \leq \beta$ be given. Then there exists a constant $C=C\left(\alpha, \alpha^{\prime}, n, T\right)$ depending only on $T, G, L, n, \alpha$ and $\alpha^{\prime}$ such that

$$
\hat{\mathbb{E}}\left[\sup _{t \in[0, T]}\left|Y_{t}\right|^{\alpha}\right] \leq C\left\{\left(\hat{\mathbb{E}}\left[|\xi|^{\alpha^{\prime}}\right]\right)^{\frac{\alpha}{\alpha^{\prime}}}+\left(\hat{\mathbb{E}}\left[\left(\int_{0}^{T} h_{s} d s\right)^{\alpha^{\prime}}\right]\right)^{\frac{\alpha}{\alpha^{\prime}}}\right\}
$$

where $h_{s}=|f(s, 0,0)|+\sum_{i, j=1}^{d}\left|g_{i j}(s, 0,0)\right|$.

Proof. From Corollary [3.9, we have

$$
\begin{aligned}
\hat{\mathbb{E}}\left[\sup _{t \in[0, T]}\left|Y_{t}\right|^{\alpha}\right] & \leq C\left(\hat{\mathbb{E}}\left[\sup _{t \in[0, T]} \hat{\mathbb{E}}_{t}\left[|\xi|^{\alpha}\right]\right]+\hat{\mathbb{E}}\left[\sup _{t \in[0, T]} \hat{\mathbb{E}}_{t}\left[\left(\int_{t}^{T} h_{s} d s\right)^{\alpha}\right]\right]\right) \\
& \leq C\left(\hat{\mathbb{E}}\left[\sup _{t \in[0, T]} \hat{\mathbb{E}}_{t}\left[|\xi|^{\alpha}\right]\right]+\hat{\mathbb{E}}\left[\sup _{t \in[0, T]} \hat{\mathbb{E}}_{t}\left[\left(\int_{0}^{T} h_{s} d s\right)^{\alpha}\right]\right]\right) .
\end{aligned}
$$

Now the conclusion follows from Theorem 2.2,

\section{Nonlinear Feynman-Kac formula}

In this section, we study the connection between the multi-dimensional $G$-BSDE and the system of fully nonlinear PDEs. Indeed, we shall show that the solution of $G$-BSDE (8) associated with stochastic differential equation driven by $G$-Brownian motion $(G$-SDE) is the unique viscosity solution to a system of fully nonlinear parabolic PDEs.

First, for any given $t \in[0, T]$ and $\eta \in L_{G}^{p}\left(\Omega_{t} ; \mathbb{R}^{k}\right), p \geq 2$, we introduce the following $G$-SDEs (recall that we always use the Einstein's convention):

$$
\left\{\begin{array}{l}
d X_{s}^{t, \eta}=b\left(s, X_{s}^{t, \eta}\right) d s+h_{i j}\left(s, X_{s}^{t, \eta}\right) d\left\langle B^{i}, B^{j}\right\rangle_{s}+\sigma\left(s, X_{s}^{t, \eta}\right) d B_{s}, \quad s \in[t, T], \\
X_{t}^{t, \eta}=\eta
\end{array}\right.
$$

where $b(s, x), h_{i j}(s, x):[0, T] \times \mathbb{R}^{k} \rightarrow \mathbb{R}^{k}$ and $\sigma(s, x):[0, T] \times \mathbb{R}^{k} \rightarrow \mathbb{R}^{k \times d}$ are deterministic continuous functions satisfying:

$\left(H_{3}\right) h_{i j}=h_{j i}$ for $1 \leq i, j \leq d$, and there exists a positive constant $L$ such that

$$
\left|b\left(t, x_{1}\right)-b\left(t, x_{2}\right)\right|+\sum_{i, j=1}^{d}\left|h_{i j}\left(t, x_{1}\right)-h_{i j}\left(t, x_{2}\right)\right|+\sum_{i=1}^{d}\left|\sigma\left(t, x_{1}\right)-\sigma\left(t, x_{2}\right)\right| \leq L\left|x_{1}-x_{2}\right| .
$$

Following [20], the equation (18) admits a unique $M_{G}^{p}\left(t, T ; \mathbb{R}^{k}\right)$-solution $X^{t, \xi}$. Moreover, it holds that

Lemma 4.1 Let $\eta, \eta^{\prime} \in L_{G}^{p}\left(\Omega_{t} ; \mathbb{R}^{k}\right)$ for some $p \geq 2$. Then there exists a constant $C$ depending on $L, G, p, k$ and $T$ such that for each $0 \leq t \leq t^{\prime} \leq T$,

(i) $\hat{\mathbb{E}}_{t}\left[\sup _{s \in[t, T]}\left|X_{s}^{t, \eta}-X_{s}^{t, \eta^{\prime}}\right|^{p}\right] \leq C\left|\eta-\eta^{\prime}\right|^{p}$;

(ii) $\hat{\mathbb{E}}_{t}\left[\sup _{s \in[t, T]}\left|X_{s}^{t, \eta}\right|^{p}\right] \leq C\left(1+|\eta|^{p}\right)$; 
(iii) $\hat{\mathbb{E}}_{t}\left[\sup _{s \in\left[t, t^{\prime}\right]}\left|X_{s}^{t, \eta}-\eta\right|^{p}\right] \leq C\left(1+|\eta|^{p}\right)\left(t^{\prime}-t\right)^{p / 2}$.

Next we consider the following $n$-dimensional $G$-BSDEs on the interval $[t, T]$ :

$$
\begin{aligned}
Y_{s}^{t, \eta ; l}= & \varphi^{l}\left(X_{T}^{t, \eta}\right)+\int_{s}^{T} f^{l}\left(r, X_{r}^{t, \eta}, Y_{r}^{t, \eta}, Z_{r}^{t, \eta ; l}\right) d r+\int_{s}^{T} g_{i j}^{l}\left(r, X_{r}^{t, \eta}, Y_{r}^{t, \eta}, Z_{r}^{t, \eta ; l}\right) d\left\langle B^{i}, B^{j}\right\rangle_{r} \\
& -\int_{s}^{T} Z_{r}^{t, \eta ; l} d B_{r}-\left(K_{T}^{t, \eta ; l}-K_{s}^{t, \eta ; l}\right), \quad 1 \leq l \leq n,
\end{aligned}
$$

where the deterministic continuous functions $\varphi^{l}: \mathbb{R}^{k} \rightarrow \mathbb{R}, f^{l}, g_{i j}^{l}=g_{j i}^{l}:[0, T] \times \mathbb{R}^{k} \times \mathbb{R}^{n} \times \mathbb{R}^{d} \rightarrow \mathbb{R}$, $1 \leq l \leq n$, satisfy the following assumptions:

$\left(H_{4}\right)$ There exists a constant $L \geq 0$ such that

$$
\begin{aligned}
& \left|\varphi^{l}\left(x_{1}\right)-\varphi^{l}\left(x_{2}\right)\right|+\left|f^{l}\left(t, x_{1}, y_{1}, z_{1}^{l}\right)-f\left(t, x_{2}, y_{2}, z_{2}^{l}\right)\right|+\sum_{i . j=1}^{d}\left|g_{i j}^{l}\left(t, x_{1}, y_{1}, z_{1}^{l}\right)-g_{i j}^{l}\left(t, x_{2}, y_{2}, z_{2}^{l}\right)\right| \\
& \leq L\left(\left|x_{1}-x_{2}\right|+\left|y_{1}-y_{2}\right|+\left|z_{1}^{l}-z_{2}^{l}\right|\right) .
\end{aligned}
$$

Theorem 4.2 Assume that $\eta \in L_{G}^{\beta}\left(\Omega_{t} ; \mathbb{R}^{k}\right)$ for some $\beta \geq 2$ and $\left(H_{3}\right)-\left(H_{4}\right)$ hold. Then, for any $1<\alpha<\beta$, the multi-dimensional $G$-BSDE (19) admits a unique solution $\left(Y^{t, \eta}, Z^{t, \eta}, K^{t, \eta}\right) \in$ $S_{G}^{\alpha}\left(t, T ; \mathbb{R}^{n}\right) \times H_{G}^{\alpha}\left(t, T ; \mathbb{R}^{n \times d}\right) \times \mathcal{A}_{G}^{\alpha}\left(t, T ; \mathbb{R}^{n}\right)$.

Proof. Since $\eta \in L_{G}^{\beta}\left(\Omega_{t} ; \mathbb{R}^{k}\right)$, we have $X^{t, \eta} \in M_{G}^{\beta}\left(t, T ; \mathbb{R}^{k}\right)$ and $X_{T}^{t, \eta} \in L_{G}^{\beta}\left(\Omega_{T} ; \mathbb{R}^{k}\right)$. From the Lipschitz continuity assumption of $\varphi$, we can find some constant $C$ such that

$$
\hat{\mathbb{E}}\left[\left|\varphi\left(X_{T}^{t, \eta}\right)\right|^{\beta} I_{\left\{\left|\varphi\left(X_{T}^{t, \eta}\right)\right|>N\right\}}\right] \leq C \hat{\mathbb{E}}\left[\left(1+\left|X_{T}^{t, \eta}\right|^{\beta}\right) I_{\left\{\left|X_{T}^{t, \eta}\right|>\frac{N}{C}-1\right\}}\right] \rightarrow 0 \text {, as } N \rightarrow \infty,
$$

which together with the fact that $\varphi$ is continuous implies that $\varphi\left(X_{T}^{t, \eta}\right) \in L_{G}^{\beta}\left(\Omega_{T} ; \mathbb{R}^{n}\right)$. By a similar analysis and recalling Theorem 4.7 in [11, we also have $f^{l}\left(\cdot, X^{t, \eta}, y, z^{l}\right), g_{i j}^{l}\left(\cdot, X^{t, \eta}, y, z^{l}\right) \in M_{G}^{\beta}(t, T)$ for each $y, z^{l}$. Now the desired result follows from Theorem 3.5

We also have the following regularity result on $Y^{t, \eta}$.

Lemma 4.3 Assume that $\eta, \eta^{\prime} \in L_{G}^{\beta}\left(\Omega_{t} ; \mathbb{R}^{k}\right)$ for some $\beta \geq 2$ and $\left(H_{3}\right)-\left(H_{4}\right)$ hold. Then there is a constant $C$ depending on $L, G, n, k$ and $T$ such that

$$
\begin{aligned}
\left|Y_{t}^{t, \eta}-Y_{t}^{t, \eta^{\prime}}\right| & \leq C\left|\eta-\eta^{\prime}\right|, \\
\left|Y_{t}^{t, \eta}\right| & \leq C(1+|\eta|) .
\end{aligned}
$$

Proof. We only prove the first inequality, since the other one can be obtained in a similar way. From Proposition 3.8, we can find some generic constant $C$ depends on $L, G, n$ and $T$ that

$$
\begin{aligned}
& \left|Y_{t}^{t, \eta}-Y_{t}^{t, \eta^{\prime}}\right|^{2} \leq C \hat{\mathbb{E}}_{t}\left[\left|\varphi\left(X_{T}^{t, \eta}\right)-\varphi\left(X_{T}^{t, \eta^{\prime}}\right)\right|^{2}+\left(\int_{t}^{T} \hat{h}_{s} d s\right)^{2}\right] \\
& \left.\leq C\left\{\hat{\mathbb{E}}_{t}\left[\left|X_{T}^{t, \eta}-X_{T}^{t, \eta^{\prime}}\right|^{2}\right]+\int_{t}^{T} \hat{\mathbb{E}}_{t}\left[\left|X_{s}^{t, \eta}-X_{s}^{t, \eta^{\prime}}\right|^{2}\right] d s\right]\right\},
\end{aligned}
$$


where

$$
\begin{aligned}
\hat{h}_{s} & =\left|f\left(s, X_{s}^{t, \eta}, Y_{s}^{t, \eta^{\prime}}, Z_{s}^{t, \eta^{\prime}}\right)-f\left(s, X_{s}^{t, \eta^{\prime}}, Y_{s}^{t, \eta^{\prime}}, Z_{s}^{t, \eta^{\prime}}\right)\right| \\
& +\sum_{i, j=1}^{d}\left|g_{i j}\left(s, X_{s}^{t, \eta}, Y_{s}^{t, \eta^{\prime}}, Z_{s}^{t, \eta^{\prime}}\right)-g_{i j}\left(s, X_{s}^{t, \eta^{\prime}}, Y_{s}^{t, \eta^{\prime}}, Z_{s}^{t, \eta^{\prime}}\right)\right| .
\end{aligned}
$$

Next recalling Lemma 4.1 we have, for $s \geq t$,

$$
\hat{\mathbb{E}}_{t}\left[\left|X_{s}^{t, \eta}-X_{s}^{t, \eta^{\prime}}\right|^{2}\right] \leq C\left|\eta-\eta^{\prime}\right|^{2} .
$$

Combining this with (20), we get the desired result.

For each $(t, x) \in[0, T] \times \mathbb{R}^{k}$, we define the following deterministic function

$$
u(t, x):=Y_{t}^{t, x}, \text { for each }(t, x) \in[0, T] \times \mathbb{R}^{k} .
$$

Then it follow from Lemma 4.3 that $u$ satisfies

$$
\left|u(t, x)-u\left(t, x^{\prime}\right)\right| \leq C\left|x-x^{\prime}\right|, \text { for all } x, x^{\prime} \in \mathbb{R}^{k} .
$$

In the following, we will show that $u$ is the viscosity solution of the following system of parabolic PDEs:

$$
\begin{cases}\partial_{t} u^{l}(t, x)+F^{l}\left(D_{x}^{2} u^{l}, D_{x} u^{l}, u, x, t\right)=0, & (t, x) \in(0, T) \times \mathbb{R}^{k}, \\ u^{l}(T, x)=\varphi^{l}(x), & x \in \mathbb{R}^{k} ; 1 \leq l \leq n,\end{cases}
$$

where

$$
\begin{aligned}
F^{l}(A, p, r, x, t) & :=G\left(\sigma^{T}(t, x) A \sigma(t, x)+2\left[\left\langle p, h_{i j}(t, x)\right\rangle\right]_{i, j=1}^{d}+2\left[g_{i j}^{l}\left(t, x, r, \sigma^{T}(t, x) p\right)\right]_{i, j=1}^{d}\right)+\langle b(t, x), p\rangle \\
& +f^{l}\left(t, x, r, \sigma^{T}(t, x) p\right), \text { for }(A, p, r, x, t) \in \mathbb{S}(k) \times \mathbb{R}^{k} \times \mathbb{R}^{n} \times \mathbb{R}^{k} \times[0, T] .
\end{aligned}
$$

Let us first give the definition of viscosity solutions of the system (23).

Definition 4.4 Let $u \in C\left([0, T] \times \mathbb{R}^{k} ; \mathbb{R}^{n}\right)$. $u$ is said to be a viscosity sub-solution of (23) if for each $1 \leq l \leq n, u^{l}(T, x) \leq \varphi^{l}(x)$ for $x \in \mathbb{R}^{k}$, and for any $(t, x) \in(0, T) \times \mathbb{R}^{k}, \psi \in C^{1,2}\left((0, T) \times \mathbb{R}^{k}\right)$ such that $\psi \geq u^{l}, \psi(t, x)=u^{l}(t, x)$, we have

$$
\partial_{t} \psi(t, x)+F^{l}\left(D_{x}^{2} \psi, D_{x} \psi, u(x, t), x, t\right) \geq 0 .
$$

$u$ is is said to be a viscosity super-solution of (23) if for each $1 \leq l \leq n, u^{l}(T, x) \geq \varphi^{l}(x)$ for $x \in \mathbb{R}^{k}$, and for any $(t, x) \in(0, T) \times \mathbb{R}^{k}, \psi \in C^{1,2}\left((0, T) \times \mathbb{R}^{k}\right)$ such that $\psi \leq u^{l}, \psi(t, x)=u^{l}(t, x)$, we have

$$
\partial_{t} \psi(t, x)+F^{l}\left(D_{x}^{2} \psi, D_{x} \psi, u(x, t), x, t\right) \leq 0 .
$$

If $u$ is both a viscosity sub-solution and a super-solution, we call $u$ a viscosity solution of (23).

Now we derive the following regularity result on $u$.

Proposition 4.5 The function $u(t, x):[0, T] \times \mathbb{R}^{k} \rightarrow \mathbb{R}^{n}$ is continuous.

Proof. For symbol simplicity, we may assume $g_{i j} \equiv 0$. For any $t \geq 0$, we define

$$
Y_{s}^{t, x}:=Y_{t}^{t, x}, X_{s}^{t, x}:=X_{t}^{t, x}=x, K_{s}^{t, x}:=0, Z_{s}^{t, x}:=0, \quad \text { for each } s \in[0, t] .
$$


Then $Y^{t, x}$ is the solution of the following $G$-BSDE on the interval $[0, T]$ :

$$
Y_{s}^{t, x ; l}=\varphi\left(X_{T}^{t, x ; l}\right)+\int_{s}^{T} \tilde{f}^{l}\left(r, X_{r}^{t, x}, Y_{r}^{t, x}, Z_{r}^{t, x ; l}\right) d r-\int_{s}^{T} Z_{r}^{t, x ; l} d B_{r}-\left(K_{T}^{t, x ; l}-K_{s}^{t, x ; l}\right), 1 \leq l \leq n,
$$

where $\tilde{f}^{l}\left(s, X_{s}^{t, x}, Y_{s}^{t, x}, Z_{s}^{t, x ; l}\right)=I_{[0, t]}(s) f^{l}\left(s, X_{s}^{t, x}, Y_{s}^{t, x}, Z_{s}^{t, x ; l}\right)$. For $0 \leq t \leq t^{\prime} \leq T$, applying Proposition 3.8, we have

$$
\begin{aligned}
& \left|u(t, x)-u\left(t^{\prime}, x\right)\right|^{2}=\left|Y_{0}^{t, x}-Y_{0}^{t^{\prime}, x}\right|^{2} \\
& \leq C \hat{\mathbb{E}}\left[\left|\varphi\left(X_{T}^{t, x}\right)-\varphi\left(X_{T}^{t^{\prime}, x}\right)\right|^{2}\right] \\
& +C \hat{\mathbb{E}}\left[\left|\int_{0}^{T} I_{[0, t]}(r) f^{l}\left(r, X_{r}^{t, x}, Y_{r}^{t, x}, Z_{r}^{t, x}\right) d r-\int_{0}^{T} I_{\left[0, t^{\prime}\right]}(r) f^{l}\left(r, X_{r}^{t, x}, Y_{r}^{t, x}, Z_{r}^{t, x}\right) d r\right|^{2}\right] \\
& +C \hat{\mathbb{E}}\left[\left|\int_{0}^{T} I_{\left[0, t^{\prime}\right]}(r) f^{l}\left(r, X_{r}^{t, x}, Y_{r}^{t, x}, Z_{r}^{t, x}\right) d r-\int_{0}^{T} I_{\left[0, t^{\prime}\right]}(r) f^{l}\left(r, X_{r}^{t^{\prime}, x}, Y_{r}^{t, x}, Z_{r}^{t, x}\right) d r\right|^{2}\right] \\
& =: I_{1}+I_{2}+I_{3} .
\end{aligned}
$$

We will estimate the above three terms separately. First note that the uniqueness theorem of $G$-SDEs implies $X_{r}^{t, x}=X_{r}^{t^{\prime}, X_{t^{\prime}}^{t, x}}$, for $r \geq t^{\prime}$. Then by Lemma 4.1, we obtain

$$
\hat{\mathbb{E}}\left[\left|X_{r}^{t, x}-X_{r}^{t^{\prime}, x}\right|^{2}\right]=\hat{\mathbb{E}}\left[\left|X_{r}^{t^{\prime}, X_{t^{\prime}}^{t, x}}-X_{r}^{t^{\prime}, x}\right|^{2}\right] \leq C \hat{\mathbb{E}}\left[\left|X_{t^{\prime}}^{t, x}-x\right|^{2}\right] \leq C\left(1+|x|^{2}\right)\left(t^{\prime}-t\right) \text {, for } r \geq t^{\prime} .
$$

From this we get

$$
I_{1} \leq C \hat{\mathbb{E}}\left[\left|X_{T}^{t, x}-X_{T}^{t^{\prime}, x}\right|^{2}\right] \leq C\left(1+|x|^{2}\right)\left(t^{\prime}-t\right) .
$$

By Proposition 3.10 and Lemma 4.1 we have

$$
\hat{\mathbb{E}}\left[\sup _{t \leq s \leq T}\left|Y_{s}^{t, x}\right|^{2}\right] \leq C\left(1+|x|^{2}\right) .
$$

Applying Proposition 3.5 in [7] to each component of (24) and using inequality (26), we then deduce that

$$
\hat{\mathbb{E}}\left[\int_{t}^{T}\left|Z_{s}^{t, x ; l}\right|^{2} d s\right] \leq C\left(\hat{\mathbb{E}}\left[\sup _{t \leq s \leq T}\left|Y_{s}^{t, x ; l}\right|^{2}\right]+\hat{\mathbb{E}}\left[\int_{t}^{T}\left|f^{l}\left(s, X_{s}^{t, x}, Y_{s}^{t, x}, 0\right)\right|^{2} d s\right]\right) \leq C\left(1+|x|^{2}\right) .
$$

In spirit of inequalities (26) and (27), we obtain

$$
\begin{aligned}
I_{2} & =C \hat{\mathbb{E}}\left[\left|\int_{t}^{t^{\prime}} f^{l}\left(r, X_{r}^{t, x}, Y_{r}^{t, x}, Z_{r}^{t, x ; l}\right) d r\right|^{2}\right] \leq C\left(\hat{\mathbb{E}}\left[\int_{t}^{T}\left|f^{l}\left(r, X_{r}^{t, x}, Y_{r}^{t, x}, Z_{r}^{t, x ; l}\right)\right|^{2} d r\right]\right)\left(t^{\prime}-t\right) \\
& \leq C\left(1+|x|^{2}\right)\left(t^{\prime}-t\right) .
\end{aligned}
$$

Applying estimate (25) again, we have

$$
\begin{aligned}
I_{3} & \leq C \hat{\mathbb{E}}\left[\int_{0}^{T} I_{\left[0, t^{\prime}\right]}(r)\left|f^{l}\left(r, X_{r}^{t, x}, Y_{r}^{t, x}, Z_{r}^{t, x}\right) d r-f^{l}\left(r, X_{r}^{t^{\prime}, x}, Y_{r}^{t, x}, Z_{r}^{t, x}\right)\right|^{2} d r\right] \\
& \leq C \hat{\mathbb{E}}\left[\int_{0}^{T} I_{\left[0, t^{\prime}\right]}(r)\left|X_{r}^{t, x}-X_{r}^{t^{\prime}, x}\right|^{2} d r\right] \leq C\left(1+|x|^{2}\right)\left(t^{\prime}-t\right) .
\end{aligned}
$$

Consequently, it holds that

$$
\left|u(t, x)-u\left(t^{\prime}, x\right)\right| \leq C(1+|x|)\left(t^{\prime}-t\right)^{1 / 2},
$$

which together with inequality (22) indicate the desired result.

We will also need the following lemma, whose proof is similar to that of Theorem 4.4 in 8$]$ and so is omitted. 
Lemma 4.6 Assume $\eta \in L_{G}^{\beta}\left(\Omega_{t} ; \mathbb{R}^{k}\right)$ for some $\beta \geq 2$. Then

$$
u(t, \eta)=Y_{t}^{t, \eta} .
$$

We now state the main result of this section.

Theorem 4.7 Assume $\left(H_{3}\right)-\left(H_{4}\right)$ hold. Then $u$ is the unique viscosity solution of the backward parabolic equation system (23).

Proof. We first prove that $u$ is a viscosity solution of (23). We only show that $u$ is a viscosity sub-solution, and the proof of sup-solution is similar.

Let $1 \leq l \leq n$ be given. Assume $\psi \in C^{1,2}\left((0, T) \times \mathbb{R}^{k}\right)$ such that $\psi \geq u^{l}, \psi(t, x)=u^{l}(t, x)$ for some $(t, x) \in(0, T) \times \mathbb{R}^{k}$. Since $u$ is Lipschitz continuous and from a standard argument, we can assume that $\psi$ is continuously differential up to the third order and the corresponding derivatives of order from 1 to 3 are bounded. We need to prove that

$$
\partial_{t} \psi(t, x)+F^{l}\left(D_{x}^{2} \psi(x, t), D_{x} \psi(x, t), u(x, t), x, t\right) \geq 0 .
$$

For $t^{\prime} \geq t$, let $(\tilde{Y}, \tilde{Z}, \tilde{K})$ be the solution the following one-dimensional $G$-BSDE on $\left[t, t^{\prime}\right]$ :

$$
\begin{aligned}
\tilde{Y}_{s} & =\psi\left(t^{\prime}, X_{t^{\prime}}^{t, x}\right)+\int_{s}^{t^{\prime}} f^{l}\left(r, X_{r}^{t, x}, Y_{r}^{t, x ; 1}, \cdots, Y_{r}^{t, x ; l-1}, \tilde{Y}_{r}, Y_{r}^{t, x ; l+1}, \cdots, Y_{r}^{t, x ; n}, \tilde{Z}_{r}\right) d r \\
& +\int_{s}^{t^{\prime}} g_{i j}^{l}\left(r, X_{r}^{t, x}, Y_{r}^{t, x ; 1}, \cdots, Y_{r}^{t, x ; l-1}, \tilde{Y}_{r}, Y_{r}^{t, x ; l+1}, \cdots, Y_{r}^{t, x ; n}, \tilde{Z}_{r}\right) d\left\langle B^{i}, B^{j}\right\rangle_{r} \\
& -\int_{s}^{t^{\prime}} \tilde{Z}_{r} d B_{r}-\left(\tilde{K}_{t^{\prime}}-\tilde{K}_{s}\right) .
\end{aligned}
$$

We first note that, from the $G$-Itô's formula (see Section III of [20]),

$$
\begin{aligned}
\psi\left(t^{\prime}, X_{t^{\prime}}^{t, x}\right) & =\psi\left(s, X_{s}^{t, x}\right)+\int_{s}^{t^{\prime}} \partial_{t} \psi\left(r, X_{r}^{t, x}\right) d r+\int_{s}^{t^{\prime}}\left\langle D_{x} \psi\left(r, X_{r}^{t, x}\right), b\left(r, X_{r}^{t, x}\right)\right\rangle d r \\
& +\int_{s}^{t^{\prime}}\left\langle D_{x} \psi\left(r, X_{r}^{t, x}\right), h_{i j}\left(r, X_{r}^{t, x}\right)\right\rangle d\left\langle B^{i}, B^{j}\right\rangle_{r}+\int_{s}^{t^{\prime}}\left\langle\sigma^{T}\left(r, X_{r}^{t, x}\right) D_{x} \psi\left(r, X_{r}^{t, x}\right), d B_{r}\right\rangle \\
& +\frac{1}{2} \int_{s}^{t^{\prime}}\left(\sigma^{T}\left(r, X_{r}^{t, x}\right) D_{x}^{2} \psi\left(r, X_{r}^{t, x}\right) \sigma\left(r, X_{r}^{t, x}\right)\right)_{i j} d\left\langle B^{i}, B^{j}\right\rangle_{r}, \quad s \in\left[t, t^{\prime}\right] .
\end{aligned}
$$

Moreover, by the uniqueness theorem of multi-dimensional $G$-BSDEs and Lemma 4.6, we have

$$
Y_{s}^{t, x}=Y_{s}^{s, X_{s}^{t, x}}=u\left(s, X_{s}^{t, x}\right), \text { for } s \geq t .
$$

Thus, if we denote $\hat{Y}_{s}=\tilde{Y}_{s}-\psi\left(s, X_{s}^{t, x}\right), \hat{Z}_{s}=\tilde{Z}_{s}-\left(\sigma^{T} D_{x} \psi\right)\left(s, X_{s}^{t, x}\right), \hat{K}_{s}=\tilde{K}_{s}$. Then $(\hat{Y}, \hat{Z}, \hat{K})$ is the solution of the following $G$-BSDE on $\left[t, t^{\prime}\right]$ :

$$
\begin{aligned}
\hat{Y}_{s}= & \int_{s}^{t^{\prime}} e^{l}\left(r, X_{r}^{t, x}, \hat{Y}_{r}, \hat{Z}_{r}\right) d r+\int_{s}^{t^{\prime}} k_{i j}^{l}\left(r, X_{r}^{t, x}, \hat{Y}_{r}, \hat{Z}_{r}\right) d\left\langle B^{i}, B^{j}\right\rangle_{r} \\
& -\int_{s}^{t^{\prime}} \hat{Z}_{r} d B_{r}-\left(\hat{K}_{t^{\prime}}-\hat{K}_{s}\right) .
\end{aligned}
$$

where

$$
\begin{aligned}
e^{l}(s, x, y, z) & =f^{l}\left(s, x, u^{1}(s, x), \cdots, u^{l-1}(s, x), y+\psi(s, x), u^{l+1}(s, x), \cdots, u^{n}(s, x), z^{l}+\left(\sigma^{T} D_{x} \psi\right)(s, x)\right) \\
& +\partial_{t} \psi(s, x)+\left\langle b(s, x), D_{x} \psi(s, x)\right\rangle,
\end{aligned}
$$




$$
\begin{aligned}
k_{i j}^{l}(s, x, y, z) & =g_{i j}^{l}\left(s, x, u^{1}(s, x), \cdots, u^{l-1}(s, x), y+\psi(s, x), u^{l+1}(s, x), \cdots, u^{n}(s, x), z^{l}+\left(\sigma^{T} D_{x} \psi\right)(s, x)\right) \\
& +\left\langle D_{x} \psi(s, x), h_{i j}(s, x)\right\rangle+\frac{1}{2}\left(\sigma^{T} D_{x}^{2} \psi \sigma\right)_{i j}(s, x) .
\end{aligned}
$$

We now consider a deterministic approximation of the above $G$-BSDE (31). Let $(\bar{Y}, \bar{Z}, \bar{K})$ be the solution of the following $G$-BSDE on $\left[t, t^{\prime}\right]$ :

$$
\bar{Y}_{s}=\int_{s}^{t^{\prime}} e^{l}\left(r, x, \bar{Y}_{r}, \bar{Z}_{r}\right) d r+\int_{s}^{t^{\prime}} k_{i j}^{l}\left(r, x, \bar{Y}_{r}, \bar{Z}_{r}\right) d\left\langle B^{i}, B^{j}\right\rangle_{r}-\int_{s}^{t^{\prime}} \bar{Z}_{r} d B_{r}-\left(\bar{K}_{t^{\prime}}-\bar{K}_{s}\right) .
$$

We first take $\bar{Z}=0$. Note that

$$
\begin{aligned}
\bar{Y}_{s} & =\int_{s}^{t^{\prime}}\left[e^{l}\left(r, x, \bar{Y}_{r}, 0\right)+2 G\left(\left[k_{i j}^{l}\left(r, x, \bar{Y}_{r}, 0\right)\right]_{i, j=1}^{d}\right)\right] d r \\
& +\int_{s}^{t^{\prime}} k_{i j}^{l}\left(r, x, \bar{Y}_{r}, 0\right) d\left\langle B^{i}, B^{j}\right\rangle_{r}-\int_{s}^{t^{\prime}} 2 G\left(\left[k_{i j}^{l}\left(r, x, \bar{Y}_{r}, 0\right)\right]_{i, j=1}^{d}\right) d r .
\end{aligned}
$$

Thus if we take $\bar{Y}_{s}$ as the solution of the following ODE:

$$
\bar{Y}_{s}=\int_{s}^{t^{\prime}}\left[e^{l}\left(r, x, \bar{Y}_{r}, 0\right)+2 G\left(\left[k_{i j}^{l}\left(r, x, \bar{Y}_{r}, 0\right)\right]_{i, j=1}^{d}\right)\right] d r
$$

and define

$$
\bar{K}_{s}:=\int_{t}^{s} k_{i j}^{l}\left(r, x, \bar{Y}_{r}, 0\right) d\left\langle B^{i}, B^{j}\right\rangle_{r}-\int_{t}^{s} 2 G\left(\left[k_{i j}^{l}\left(r, x, \bar{Y}_{r}, 0\right)\right]_{i, j=1}^{d}\right) d r,
$$

then it is easy to see that $(\bar{Y}, 0, \bar{K})$ is the solution of (32). Applying Proposition 2.7 and Lemma 4.1 . we have

$$
\left|\hat{Y}_{t}-\bar{Y}_{t}\right|^{2} \leq C \hat{\mathbb{E}}_{t}\left[\left(\int_{t}^{t^{\prime}} \hat{h}_{r} d r\right)^{2}\right] \leq C\left(\int_{t}^{t^{\prime}} \hat{\mathbb{E}}_{t}\left[\left|\hat{h}_{r}\right|^{2}\right] d r\right)\left(t^{\prime}-t\right) \leq C\left(1+|x|^{2}\right)\left(t^{\prime}-t\right)^{3},
$$

where

$$
\hat{h}_{r}=\left|e^{l}\left(r, X_{r}^{t, x}, \bar{Y}_{r}, 0\right)-e^{l}\left(r, x, \bar{Y}_{r}, 0\right)\right|+\sum_{i, j=1}^{d}\left|k_{i j}^{l}\left(r, X_{r}^{t, x}, \bar{Y}_{r}, 0\right)-k_{i j}^{l}\left(r, x, \bar{Y}_{r}, 0\right)\right| .
$$

Therefore,

$$
\left|\hat{Y}_{t}-\bar{Y}_{t}\right| \leq C(1+|x|)\left(t^{\prime}-t\right)^{\frac{3}{2}}
$$

Moreover, in view of the assumption on $\psi$ and applying Theorem 2.6 to $G$-BSDEs (30) and

$$
\begin{aligned}
Y_{s}^{t, x ; l}= & u^{l}\left(t^{\prime}, X_{t^{\prime}}^{t, x}\right)+\int_{s}^{t^{\prime}} f^{l}\left(r, X_{r}^{t, x}, Y_{r}^{t, x}, Z_{r}^{t, x ; l}\right) d r+\int_{s}^{t^{\prime}} g_{i j}^{l}\left(r, X_{r}^{t, x}, Y_{r}^{t, x}, Z_{r}^{t, x ; l}\right) d\left\langle B^{i}, B^{j}\right\rangle_{s} \\
& -\int_{s}^{t^{\prime}} Z_{r}^{t, x ; l} d B_{r}-\left(K_{t^{\prime}}^{t, x ; l}-K_{s}^{t, x ; l}\right), t \leq s \leq t^{\prime},
\end{aligned}
$$

we get

$$
\tilde{Y}_{t} \geq u^{l}(t, x)
$$

This implies

$$
\hat{Y}_{t} \geq 0 .
$$


Combining this with (34), we obtain

$$
\left(t^{\prime}-t\right)^{-1} \bar{Y}_{t} \geq-C(1+|x|)\left(t^{\prime}-t\right)^{1 / 2} .
$$

That is,

$$
\left(t^{\prime}-t\right)^{-1} \int_{t}^{t^{\prime}}\left[e^{l}\left(r, x, \bar{Y}_{r}, 0\right)+2 G\left(\left[k_{i j}^{l}\left(r, x, \bar{Y}_{r}, 0\right)\right]_{i, j=1}^{d}\right)\right] d r \geq-C(1+|x|)\left(t^{\prime}-t\right)^{1 / 2} .
$$

On the other hand, note that from (33),

$$
\left|\bar{Y}_{s}\right| \leq C \int_{s}^{t^{\prime}}\left(1+\left|\bar{Y}_{r}\right|\right) d r \leq C\left(\left(t^{\prime}-t\right)+\int_{s}^{t^{\prime}}\left|\bar{Y}_{r}\right|\right) d r, \quad s \in\left[t, t^{\prime}\right]
$$

which implies, by the Gronwall's inequality,

$$
\left|\bar{Y}_{s}\right| \leq C\left(t^{\prime}-t\right), \quad s \in\left[t, t^{\prime}\right]
$$

Therefore, letting $t^{\prime} \rightarrow t$ in (35), we get

$$
e^{l}(t, x, 0,0)+2 G\left(\left[k_{i j}^{l}(r, x, 0,0)\right]_{i, j=1}^{d}\right) \geq 0,
$$

which is just (29) and this means that $u$ is a viscosity sub-solution.

Analysis similar to the proof of Theorem 5.1 in [15] (see also [1, 2, 3]) shows that there exists at most one solution to the system (23) in the class of continuous functions with polynomially growth. The proof is complete.

Acknowledgement: The author is grateful to Dr. Mingshang Hu and Dr. Falei Wang for their fruitful discussions. The author would also like to thank the anonymous referee for the valuable comments and constructive suggestions which improved the presentation of this manuscript.

\section{References}

[1] Barles, G., Buckdahn, R. and Pardoux, E. Backward stochastic differential equations and integral-partial differential equations. Stochastics Stochastics Rep. 60 (1997), no. 1-2, 57-83.

[2] Buckdahn, R. and Hu, Y. Probabilistic interpretation of a coupled system of Hamilton-Jacobi-Bellman equations. J. Evol. Equ. 10 (2010), no. 3, 529-549.

[3] Buckdahn, R. and Li, J. Stochastic differential games and viscosity solutions of Hamilton-Jacobi-BellmanIsaacs equations. SIAM J. Control Optim. 47 (2008), no. 1, 444-475.

[4] Coquet, F., Hu, Y., Memin J. and Peng, S. Filtration consistent nonlinear expectations and related g-expectations. Probab. Theory Relat. Fields 123 (2002), no. 1, 1-27.

[5] Denis, L., Hu, M. and Peng, S. Function spaces and capacity related to a sublinear expectation: application to G-Brownian motion paths. Potential Anal. 34 (2011), no. 2, 139-161.

[6] El Karoui, N., Peng, S. and Quenez, M. C. Backward stochastic differential equations in finance. Math. Finance, 7 (1997), no. 1, 1-71.

[7] Hu, M., Ji, S., Peng, S. and Song, Y. Backward stochastic differential equations driven by G-Brownian motion. Stochastic Process. Appl. 124 (2014), no. 1, 759-784. 
[8] Hu, M., Ji, S., Peng, S. and Song, Y. Comparison theorem, Feynman-Kac formula and Girsanov transformation for BSDEs driven by G-Brownian motion. Stochastic Process. Appl. 124 (2014), no. 2, 1170-1195.

[9] Hu, M. and Peng, S. On representation theorem of $G$-expectations and paths of $G$-Brownian motion. Acta Math. Appl. Sin. Engl. Ser. 25 (2009), no. 3, 539-546.

[10] $\mathrm{Hu}, \mathrm{M}$. and Wang, F. Ergodic BSDEs driven by G-Brownian motion and applications. Stoch. Dyn. 18 (2018), no. 6, 1-35.

[11] Hu, M., Wang, F. and Zheng, G. Quasi-continuous random variables and processes under the Gexpectation framework. Stochastic Process. Appl. 126 (2016), no. 8, 2367-2387.

[12] Li, H., Peng, S. and Hima, A. S. Reflected solutions of backward stochastic differential equations driven by $G$-Brownian motion. Sci. China Math. 61 (2018), no. 1, 1-26.

[13] Pardoux, E. and Peng, S. Adapted solution of a backward stochastic differential equation. Systems Control Lett., 14 (1), 55-61, 1990.

[14] Pardoux, E. and Peng, S. Backward stochastic differential equations and quasilinear parabolic partial differential equations. Stochastic partial differential equations and their applications, 200-217, Lect. Notes Control Inf. Sci., 176, Springer, Berlin, 1992.

[15] Pardoux, E., Pradeilles, F. and Rao, Z. Probabilistic interpretation of a system of semi-linear parabolic partial differential equations. Ann. Inst. H. Poincaré Probab. Statist. 33 (1997), no. 4, 467-490.

[16] Peng, S. Probabilistic interpretation for systems of quasilinear parabolic partial differential equations. Stochastics Stochastics Rep. 37 (1991), no. 1-2, 61-74.

[17] Peng, S. Backward stochastic differential equations and applications to optimal control. Appl. Math. Optim. 27 (1993), no. 2, 125-144.

[18] Peng, S. G-expectation, G-Brownian motion and related stochastic calculus of Itô type. Stochastic analysis and applications, 541-567, Abel Symp., 2, Springer, Berlin, 2007.

[19] Peng, S. Multi-dimensional $G$-Brownian motion and related stochastic calculus under $G$-expectation. Stochastic Process. Appl. 118 (2008), no. 12, 2223-2253.

[20] Peng, S. Nonlinear expectations and stochastic calculus under uncertainty. ArXiv:1002.4546v1, 2010.

[21] Soner, H. M., Touzi, N. and Zhang, J. Wellposedness of Second Order Backward SDEs. Probab. Theory Related Fields 153 (2012), no. 1-2, 149190.

[22] Song, Y. Some properties on $G$-evaluation and its applications to $G$-martingale decomposition. Sci. China Math. 54 (2011), no. 2, 287-300.

[23] Song, Y. Properties of $G$-martingales with finite variation and the application to $G$-Sobolev spaces. To appear in Stochastic Process. Appl. 